\title{
Shisa6 mediates cell-type specific regulation of depression in the nucleus accumbens
}

\author{
Hee-Dae Kim ${ }^{1}$, Jing Wei ${ }^{1}$, Tanessa Call ${ }^{1}$, Nicole Teru Quintus ${ }^{1}$, Alexander J. Summers ${ }^{1}$, Samantha Carotenuto ${ }^{1}$, Ross Johnson ${ }^{1}$,
} Xiaokuang $\mathrm{Ma}\left(\mathbb{D}^{1}{ }^{1}\right.$, Chenxi $\mathrm{Xu}^{2}$, Jin G. Park (iD ${ }^{2}$, Shenfeng Qiu ${ }^{1}$ and Deveroux Ferguson ${ }^{1 凶}$

(c) The Author(s) 2021

\begin{abstract}
Depression is the leading cause of disability and produces enormous health and economic burdens. Current treatment approaches for depression are largely ineffective and leave more than $50 \%$ of patients symptomatic, mainly because of non-selective and broad action of antidepressants. Thus, there is an urgent need to design and develop novel therapeutics to treat depression. Given the heterogeneity and complexity of the brain, identification of molecular mechanisms within specific cell-types responsible for producing depression-like behaviors will advance development of therapies. In the reward circuitry, the nucleus accumbens (NAc) is a key brain region of depression pathophysiology, possibly based on differential activity of D1- or D2- medium spiny neurons (MSNs). Here we report a circuit- and cell-type specific molecular target for depression, Shisa6, recently defined as an AMPAR component, which is increased only in D1-MSNs in the NAc of susceptible mice. Using the Ribotag approach, we dissected the transcriptional profile of D1- and D2-MSNs by RNA sequencing following a mouse model of depression, chronic social defeat stress (CSDS). Bioinformatic analyses identified cell-type specific genes that may contribute to the pathogenesis of depression, including Shisa6. We found selective optogenetic activation of the ventral tegmental area (VTA) to NAc circuit increases Shisa6 expression in D1-MSNs. Shisa6 is specifically located in excitatory synapses of D1-MSNs and increases excitability of neurons, which promotes anxiety- and depression-like behaviors in mice. Cell-type and circuit-specific action of Shisa6, which directly modulates excitatory synapses that convey aversive information, identifies the protein as a potential rapid-antidepressant target for aberrant circuit function in depression.
\end{abstract}

Molecular Psychiatry (2021) 26:7316-7327; https://doi.org/10.1038/s41380-021-01217-8

\section{INTRODUCTION}

Major depressive disorder (MDD) is a leading cause of disability with few effective treatments. More than 300 million individuals worldwide suffer from depression [1]. In the U.S., 30 million individuals suffer from depression, which accounts for up to $\$ 60$ billion dollars lost in annual productivity [2]. Current treatment approaches for depression are largely ineffective and leave more than $50 \%$ of patients symptomatic [3-5]. The development of novel antidepressant medications has been stagnant over the past several decades, with a majority of antidepressants derived from classic monoaminergic mechanisms serendipitously discovered more than half a century ago [6]. Thus, there is an urgent need to design and develop novel therapeutics to treat depression. Given the heterogeneity and complexity of the brain, identification of molecular mechanisms within specific cell-types responsible for producing depression-like behaviors will advance development of therapies for this disorder.

A significant challenge of gene expression analysis in whole brain tissue is that changes cannot be linked to a single cell-type, and gene expression changes that are opposing in different celltypes can be difficult to detect because these changes are averaged across whole populations [7]. Cell-type specific analysis using the RiboTag (RT) approach provides a higher level of resolution and selectivity required to address these limitations by providing actively translated transcriptomic information $[8,9]$. The nucleus accumbens (NAc), a key brain reward region, is most often associated with rewarding and motivational effects, which are reduced in depression. More than $95 \%$ of neurons in the NAc are primarily composed of two medium spiny neuronal (MSN) subtypes enriched with dopamine D1 or D2 receptors, which have opposing roles in reward, action-value, reinforcement, and responses to drugs of abuse [10-12]. Specifically, the RT approach is advantageous for gene expression analysis of adjacent, morphologically indistinguishable D1- and D2-MSNs in the NAc. Using the RT approach, we dissected the transcriptional profile of both MSNs following chronic social defeat stress (CSDS) model of depression. Results from this study revealed that Shisa6 contributes to the cell-type-specific molecular genetic pathogenesis of depression.

\section{MATERIALS AND METHODS \\ Animals}

Male C57BL/6J mice (7-9 weeks old) were obtained from Jackson Laboratory and housed on a $12 \mathrm{~h}$ light-dark cycle with access to food and water ad libitum. Male CD1 retired breeder mice (9-13 months old)

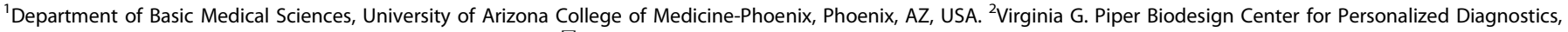
Biodesign Institute, Arizona State University, Tempe, AZ, USA. ${ }^{凶}$ email: dferguson@arizona.edu 
were obtained from Charles River Laboratories. Mice acclimated to the facility for 1 week before any experimentation. D1-Cre hemizygote (line FK150) or D2-Cre hemizygote (line ER44) BAC transgenic mice from GENSAT [13] on a C57BL/6J background were used for behavioral experiments. To induce Rpl22-HA in either D1 or D2 neurons, we crossed homozygous RT mice [8] with D1 or D2 heterozygous mice. To label D1 neurons for electrophysiological recordings, Ai6 mice were crossed with D1-Cre mice (Ai6; D1-Cre). All behavioral tests and animal sacrifices (tissue collections and acute brain slice preparations) were performed at ZT 02-06. All animal procedures were approved by the University of Arizona Medical School Institutional Animal Care and Use Committees.

\section{Chronic social defeat stress}

Social defeat stress was performed according to published protocols $[14,15]$. Test mice were exposed to an unfamiliar and aggressive $(<1 \mathrm{~min}$ latency to start aggressive behaviors) male CD1 retired breeder mouse for $5 \mathrm{~min}$ per day for up to 10 days. Following direct interaction with the CD1 aggressor, animals were placed in an adjacent compartment of the same cage for the next $24 \mathrm{~h}$ with sensory, but not physical contact. Control animals were housed in equivalent cages but with members of the same strain. $24 \mathrm{~h}$ following the last social defeat, animals were assayed on the social interaction (SI) test and sorted into either susceptible or unsusceptible (resilient) phenotypes based on interaction scores. Briefly, for the SI test in the first 2.5 min trial ("target absent"), the test mouse was allowed to explore freely a square-shaped open-field arena $(44 \times 44 \mathrm{~cm})$ possessing an empty wire-mesh cage $(10 \times 6 \mathrm{~cm})$ opposed to one side. During the second $2.5 \mathrm{~min}$ trial ("target present"), the mouse was reintroduced into this arena now containing an unfamiliar CD1 mouse within the smaller cage. EthoVision videotracking software (Noldus, Leesburg, VA) was employed to measure time spent in the "interaction zone" (IZ) $(14 \times 26 \mathrm{~cm})$. The SI ratio was calculated as $100 \times($ IZ time of "target present")/(IZ time of "target absent") [14-16]. Mice showing $<100 \%$ of SI ratio are grouped as depression-susceptible mice, and mice showing over 100\% SI ratio belong to depression-resilient group. For the transcriptome analysis, we identified distinct resilient (Res) and susceptible (Sus) mice from several batches of CSDS considering both IZ and corner zone time (CZ) (Res: increased IZ/decreased CZ, Sus: decreased IZ/increased CZ in the presence of "target" compared to "no target" session).

\section{Sub-maximal social defeat stress}

To test whether an experimental manipulation might potentiate an animal's susceptibility to defeat stress, we used a sub-maximal social defeat procedure, in which mice were subjected to repeated defeat episodes over the course of 1 day [15, 16]. Animals were placed into the CD1 aggressor's cage for $5 \mathrm{~min}$, followed by a $15 \mathrm{~min}$ break. This was repeated two further times with different CD1 aggressors.

\section{RiboTag immunoprecipitation}

Six NAc tissue punches $(2 \mathrm{~mm}$ ) from each RT; D1-Cre or RT; D2-Cre mouse are homogenized in $1 \mathrm{ml}$ of homogenization buffer [8] for each sample (two mice, which have similar SI ratio, pooled for each sample). Samples are then centrifuged at $4^{\circ} \mathrm{C}, 10,000 \mathrm{~g}$ for $10 \mathrm{~min}$ and $800 \mu \mathrm{l}$ of clear supernatants are mixed with $5 \mu \mathrm{g}$ of HA-antibody (Abcam, Cambridge, $M A)$, and incubated on a gentle rotator at $4{ }^{\circ} \mathrm{C}$ overnight. The next day, $200 \mu \mathrm{l}$ of magnetic beads (Dynabeads anti-rabbit lgG; Life technology, Carlsbad, CA) are added and incubated overnight with constant rotation. The following day, magnetic beads are washed three times in the magnetic rack for $10 \mathrm{~min}$ with a high salt wash buffer. Each RNA sample was extracted using the Direct-zol miniprep kit (Zymo Research, Irvine, CA) and subjected to qRT-PCR or RNA-seq. For quality control, RNA integrity (RIN) values were measured and samples with $\mathrm{RIN}<7$ were excluded.

\section{Library preparation and RNA-seq}

RNA-seq was performed by UAGC (University of Arizona Genetics Core, University of Arizona, Tucson, AZ). Briefly, purified RNA samples from RT immunoprecipitation (500 ng of RNA for each) were prepped for libraries after poly-A selection (KAPA Stranded mRNA-Seq Kit; Roche, Indianapolis, IN) and the libraries were sequenced in NextSeq500 (Illumina, San Diego, CA). Randomly chosen samples were divided into four sets and run in high output mode (maximum 400 million reads, $2 \times 150$ bp paired end reads) for each set. All the samples were normalized before pooling and loading on the sequencer.

\section{RNA-seq data analysis}

Data were analyzed by Rosalind (https://rosalind.onramp.bio/), with a HyperScale architecture developed by OnRamp Biolnformatics, Inc. (San Diego, CA). Reads were trimmed using cutadapt. Quality scores were assessed using FastQC. Reads were aligned to the Mus musculus genome (mm10) using STAR. Individual sample reads were quantified using HTseq and normalized via Relative Log Expression (RLE) using DESeq2 R library. Read distribution percentages, violin plots, identity heatmaps, and sample MDS plots were generated as part of the QC step using RSeQC. DEseq2 was also used to calculate fold changes and $p$ values. Clustering of genes for the final heatmap of differentially expressed genes (DEGs) was done using the Partitioning Around Medoids (PAM) method using the fpc R library. Functional enrichment analysis of pathways, gene ontology, domain structure, and other ontologies was performed using HOMER. Several database sources were referenced for enrichment analysis, including Interpro, NCBI, KEGG, MSigDB, REACTOME, WikiPathways. Enrichment was calculated relative to a set of background genes relevant to the experiment. Additional gene enrichment analyses were performed using Enrichr [17]. Heatmaps were generated with Orange (version 3.20.1) and additional statistical analyses were performed using Prism (version 8.3.0; GraphPad Software). Meta-analysis between different datasets was performed using Galaxy. The sequencing data were imported to the Galaxy web platform, and the public server at usegalaxy.org was used to analyze the data.

\section{Immunohistochemistry}

Mice were given a lethal dose of Euthasol (Virbac, Westlake, TX), then sequentially perfused with PBS and $4 \%$ paraformaldehyde. For eGRASP experiments, brains were fixed 8 weeks after viral injection to ensure robust expression of viral vectors at the nerve ends in the NAc. Brains were post-fixed in $4 \%$ paraformaldehyde overnight and cryoprotected in $30 \%$ sucrose solution at $4^{\circ} \mathrm{C}$. After equilibration in the sucrose solution, brains were sectioned at $30 \mu \mathrm{m}$ on a sliding block microtome (American Optical). Free-floating sections were washed with PBS, then blocked (3\% donkey serum, $0.1 \%$ Triton $\mathrm{X}-100$ in PBS). The following primary antibodies were applied overnight at $4{ }^{\circ} \mathrm{C}$ with constant agitation: anti-CRE (mouse monoclonal, 1:500; Millipore, Burlington, MA), anti-HA (rabbit polyclonal, 1:2000; Abcam), anti-NeuN (mouse monoclonal, 1:500; Millipore), anti-GFAP (mouse monoclonal, 1:1000; Santa Cruz, Dallas, TX), and anti-2A peptide (mouse monoclonal, 1:200; Millipore). After $4^{\circ} \mathrm{C}$ overnight incubation with constant agitation, sections were incubated with appropriate secondary antibodies conjugated with fluorescent dyes (Alexa 488, Alexa 555, and Cy5; Life technology) for $2 \mathrm{~h}$ at room temperature, after which they were washed, sections were mounted with Vectashield containing DAPI (Vector Labs, Burlingame, CA) and analyzed using confocal microscopy at various magnifications (LSM710; Carl Zeiss, San Diego, CA).

\section{In situ hybridization}

Mice were given a lethal dose of Euthasol (Virbac), then sequentially perfused with PBS and 4\% paraformaldehyde. Brains were post-fixed in 4\% paraformaldehyde overnight and cryoprotected in $30 \%$ sucrose solution at $4{ }^{\circ} \mathrm{C}$. After equilibration in the sucrose solution, brains were sectioned at $15 \mu \mathrm{m}$ on a sliding block microtome (American Optical). Free-floating sections were mounted on slides and baked for $1 \mathrm{~h}$ at $60^{\circ} \mathrm{C}$. Probes were hybridized for $2 \mathrm{~h}$ at $40^{\circ} \mathrm{C}$. Amplification steps were followed by manufacturer's instruction (RNAscope; ACD, Newark, CA). Samples were counterstained with DAPI and covered with Vectashield mounting solution (Vector Labs). Signals were imaged using confocal microscopy at various magnifications (LSM710; Carl Zeiss) and processed by Fiji (ImageJ; NIH, Bethesda, MD). For colocalization and expression analyses of Shisa6 in D1MSNs, all images were binary transformed and noise reduced by "Despeckle" and "Remove outlier" functions in ImageJ. Each corresponding Drd1 image was used as a region of interest (ROl; boundaries of D1neurons) template to calculate cell-type specific expression using the "Analyze particles" function. The total Shisa6 fluorescence signal in the ROI is equivalent to the total Shisa6 expression level of Shisa6 ${ }^{+} \mathrm{Drd}^{+}{ }^{+}$cells. Collected data from ImageJ were analyzed for two criteria: \% colocalization $=$ number of Shisa6 ${ }^{+} \mathrm{Drd}^{+}$cells/number of $\mathrm{Drd}^{+}{ }^{+}$cells, average expression level of Shisa6 ${ }^{+}$Drd $1^{+}$cells $=$total expression level of ROI/ number of Shisa6 ${ }^{+} \mathrm{Drd}^{+}$cells.

Adeno-associated viruses (AAVs) and stereotaxic surgery Cre-dependent AAV vectors (serotype 5; double inverted open reading frame (DIO) AAV construct) expressing mouse Shisa6, mCherry or shRNAs 
(for Shisa6 or scrambled) were cloned and packaged by VectorBuilder (Chicago, IL): AAV-DIO-Shisa6-T2A-mCherry $\left(6.55 \times 10^{13}(\mathrm{gc} / \mathrm{ml})\right)$, AAV-DIOmCherry $\left(2.33 \times 10^{13}\right)$, AAV-DIO-Shisa6 shRNA $\left(4.56 \times 10^{13}\right)$ and AAV-DIOscrambled $\left(1.95 \times 10^{13}\right)$. The Shisa6 knock-down target sequences are as follows: shRNA \#1, 5'-GCCCACTTGCCTCCATCATAT-3' (1117-1137); shRNA \#2, 5'-AGTATAATCATCCGATCTTAA-3' (989-1009); shRNA \#3, 5'GGTGGAGCAGTTACACTATAT-3' (1668-1688). Mice at the age of 7 weeks were anesthetized with a ketamine/xylazine mixture (ketamine $100 \mathrm{mg} / \mathrm{kg}$ and xylazine $10 \mathrm{mg} / \mathrm{kg}$ ) and prepared for stereotaxic surgery. Thirty-three gauge syringe needles (Hamilton) were used to bilaterally infuse $0.5 \mu \mathrm{l}$ of AAVs into the NAC (AP: 1.5, ML: 1.5, DV: -4.4 , angle: $\left.10^{\circ}\right)$. For the eGRASP experiments, two different pre-eGRASP (cyan and yellow) and one posteGRASP were obtained from Addgene (\#111579, 111580, and 111581) and packaged in AAV vectors (serotype 5, titers: $2.06 \times 10^{11}, 2.01 \times 10^{11}$, and $1.33 \times 10^{11}$, respectively) by VectorBuilder. The cyan pre-eGRASP and yellow pre-eGRASP AAVs $(0.5 \mu \mathrm{l}$, each site) were injected bilaterally in mPFC (AP: 1.7, ML: 0.75, DV: -2.5 , angle: $15^{\circ}$ ) and vHIP (AP: $-3.7, \mathrm{ML}: 3.0$, DV: -4.8 , angle: $\left.0^{\circ}\right)$, respectively. The post-eGRASP AAV was injected in the NAc (AP: 1.5, ML: 1.5, DV: -4.4 , angle: $10^{\circ}$ ) bilaterally (AAV-DIO-Shisa6-T2AmCherry was injected together in case of Shisa6 colabeling eGRASP experiments). For optogenetic activation of VTA afferent inputs in the NAc, retroAAV (rAAV)-Cre (serotype $\mathrm{rg}, 7 \times 10^{12}$ ) and AAV-DIO-ChR2(H134R)EYFP (or -EYFP control; serotype 5, ChR2: $4.8 \times 10^{12}$, EYFP: $6.5 \times 10^{12}$; UNC Vector Core, Chapel Hill, NC) were injected in the NAC and VTA, respectively (NAc, AP: $1.5, \mathrm{ML}: 1.5$, DV: -4.4 , angle: $10^{\circ}$; VTA, AP: -3.2 , ML: 1.0, DV: -4.6 , angle: $7^{\circ}$ ). Eight weeks after the viral injection, cannulae (custom made; LC $1.25 \mathrm{~mm}$ OD multimode ceramic zirconia ferrules (PFP), optic fiber (ThorLabs, Newton, NJ): 105 um core, $0.22 \mathrm{NA}$, Low-OH, $400-2400 \mathrm{~nm}$ ) were implanted bilaterally in the NAc (AP: 1.5, ML: 1.5, DV: -4.0 , angle: $10^{\circ}$ ) and permanently fixed to the skull with Loctite skull adhesive (Henkel, Westlake, OH). After adhesive drying, cannulas were permanently secured to the skull with Metabond (Parkell, Edgewood, NY) and dental cement (Lang Dental, Wheeling, IL). A 3\% hydrogen peroxide solution was used as a wound antiseptic.

\section{Electrophysiological recording and morphological analysis}

Whole cell patch clamp recording was conducted in D1 type medium spiny neurons (MSNs) in the NAc, followed by post-hoc neuron morphometric analysis. To overexpress Shisa6 in D1 type of MSNs, AAV-DIO-Shisa6-T2AmCherry or control AAV-DIO-mCherry were injected into the NAC (AP: 1.5, ML: $1.5, \mathrm{DV}:-4.4$, angle: $10^{\circ}$ ) bilaterally into the Ai6; D1-Cre mice. Three weeks after injection, mice were sacrificed and the brains were quickly dissected. $350 \mu \mathrm{m}$ coronal slices were made at the level of NAc on a vibratome (VT1200S; Leica, Allendale, NJ) in ice-cold ACSF (containing in $\mathrm{mM}, 126 \mathrm{NaCl}, 2.5 \mathrm{KCl}, 26 \mathrm{NaHCO}_{3}, 2 \mathrm{CaCl}_{2}, 1 \mathrm{MgCl}_{2}, 1.25 \mathrm{NaH}_{2} \mathrm{PO}_{4}$, and 10 glucose saturated with $95 \% \mathrm{O}_{2}$ and $5 \% \mathrm{CO}_{2}$ ). Slices were then incubated at $32{ }^{\circ} \mathrm{C}$ for $30 \mathrm{~min}$ before transferred to a customized recording chamber that is mounted on a microscope (BX-51Wl; Olympus, Center Valley, PA) equipped with water immersion objective (60X, NA 0.9). AAV-transduced MSNs were visualized under brightfield DIC and epifluorescence illumination. To target MSNs for whole cell recordings, neurons with soma at least $50 \mu \mathrm{m}$ below the slice surface were selected to minimize truncation of dendritic structure. The internal electrode solution contains (in $\mathrm{mM}$ ): $130 \mathrm{~K}$ gluconate, 10 HEPES, 4 KCl, 0.3 GTP-Na, 4 ATP-Mg, 2 NaCl,1 EGTA and 14 phosphocreatine ( $\mathrm{pH} 7.2,295-300 \mathrm{mOsm})$ when used for spontaneous EPSCs recordings and for action potentials evoked by current steps. The internal electrode solution for spontaneous IPSCs recording contains (in $\mathrm{mM}): 125 \mathrm{KCl}, 10$ HEPES, $2 \mathrm{MgCl}_{2}, 0.3$ GTP-Na, 2 ATP-Mg, $2.8 \mathrm{NaCl}$ and 0.6 EGTA (pH 7.2, 295-300 mOsm). Spontaneous IPSCs were recorded in ACSF supplemented with CNQX (20 M; Tocris, Minneapolis, MN). To record stimulus-evoked synaptic responses and quantify the AMPA/NMDA receptor current ratio, the electrode internal solution contains (in $\mathrm{mM}$ ): $125 \mathrm{Cs}$-gluconate, 5 TEA-Cl, $10 \mathrm{HEPES}, 2.5 \mathrm{CsCl}, 8 \mathrm{NaCl}, 5 \mathrm{QX} 314-\mathrm{HCl}, 4 \mathrm{Mg}^{2}$ ${ }_{\text {-ATP, }} 0.3 \mathrm{Na}_{3} \mathrm{GTP}, 1$ EGTA and 10 phosphocreatine. In addition, $50 \mu \mathrm{M}$ picrotoxin was added to the perfusate ACSF to block inhibitory currents. Monosynaptic responses were obtained while MSN neurons were voltage clamped at $-70 \mathrm{mV}$ (AMPAR-mediated synaptic currents) or $+40 \mathrm{mV}$ (AMPAR + NMDAR). A bipolar stimulating electrode (FHC) was placed at $\sim 100 \mathrm{~mm}$ away from the soma, and a stimulus isolator (Iso-flex, AMPI) was used to deliver a bipolar electrical stimulus.

To visualize the morphology of the recorded MSN neurons, $0.15 \%(\mathrm{~W} / \mathrm{V})$ biocytin was included in the electrode internal solution. Neuronal signal was amplified using a MultiClamp 700B patch clamp amplifier (Molecular Devices, Forster City, CA), low-pass filtered at $1 \mathrm{kHz}$ (voltage clamp) or
$10 \mathrm{kHz}$ (current clamp) and digitized at $20 \mathrm{kHz}$ using a Digidata $1440 \mathrm{~A}$ board under control of pClamp 10.6 (Molecular Devices, San Jose, CA). Only neurons in the NAc shell, which were double labeled with both ZsGreen 1 and mCherry, were recorded. To test voltage responses to current injections, incremental current steps (-100 to $300 \mathrm{pA}$ in 50pA increment) was used. To reconstruct the morphology of the recorded MSNs, slices were fixed in 4\% PFA overnight, and incubated in avidin-Alexa 488 (Life technology) for $24 \mathrm{~h}$ in PBS containing $0.2 \%$ Triton $\mathrm{X}-100$. Slices were then extensively washed, mounted on slides with two $350 \mu \mathrm{m}$-thick spacers to prevent collapsing the tissue. Dendritic arborization was visualized on a microscope (Axophot; Carl Zeiss) equipped with fluorescent light source. Neurites were traced in three-dimension using Neurolucida 360 (MicroBrightField, Williston, VT) system. After the entire arborization was traced, morphometric analysis was conducted using Neurolucida Explorer. Sholl analysis was performed to quantify the dendritic length and number of intersections at various distances from soma.

\section{Optogenetic stimulation}

Optic fiber implanted mice expressing optogenetic tools (AAV-DIO-ChR2 or -EYFP control) are prepared in advance (see AAVs and stereotaxic surgery). Chronic optogenetic stimulation started a week after the cannulation surgery. Optic fiber patch cords (ThorLabs; optic fiber: 105 um core, 0.22 $\mathrm{NA}$, Low-OH, 400-2400 nm) attached with implanted optic cannulas and rotary joint (ThorLabs; to ensure free movement of mice) are connected through an FC/PC adaptor to a $473 \mathrm{~nm}$ blue laser diode (Opto Engine LLC, Midvale, UT) and light pulses are generated through a waveform generator (Agilent, Santa Clara, CA). Laser power is controlled to be $3-5 \mathrm{~mW}$ from the tip of the fiber. Subject mice were placed in their home cages and blue light $(473 \mathrm{~nm})$ phasic pulses with $20 \mathrm{~Hz}$ for $40 \mathrm{~ms}$ were delivered for $5 \mathrm{~min}$ per day for 10 days.

\section{Behavior tests}

All subject mice were placed in the testing room $1 \mathrm{~h}$ before testing for acclimation. Data were analyzed in time bins of $2.5 \mathrm{~min}$ (1 st and 2nd half; labeled) and full time $(5 \mathrm{~min})$ and significant results were presented (otherwise, full time results were presented).

Open-field test. Open-field assessments, which are thought to reflect anxiety-related behavior, were conducted in arenas similar to those used for the SI tests (without small cage enclosures) under red light. EthoVision video tracking-based methods (Noldus) were used to record the distance traveled and the time spent in the open arena and a delineated "center zone".

Elevated-plus maze (EPM). The elevated-plus maze, an assay of anxietylike behavior, consisted of two opposite open arms $(60 \times 15 \mathrm{~cm})$ and two enclosed arms $(60 \times 15 \mathrm{~cm}$, surrounded by a $15 \mathrm{~cm}$-high black wall) elevated $75 \mathrm{~cm}$ from the ground. Animals were placed at the center of the maze for each individual trial lasting 5 min under red light. Number of entries and time spent in open arms was measured in addition to the number of entries in enclosed arms.

Light-dark box test (LDT). The light-dark box tests, an assay of anxiety-like behavior, were conducted in the same arenas used for the open-field test. A closed black box $(44 \times 15 \times 30 \mathrm{~cm})$ which has a small open door $(7 \times$ $7 \mathrm{~cm}$ ), was placed in one side of the arena. Animals were placed in the light compartment and the time spending in the light compartment were analyzed with EthoVision for a period of $5 \mathrm{~min}$. The experiment was performed under white light conditions.

Forced swim test (FST). The forced swim test, which measures despair-like behaviors, was performed as described previously [16]. Animals were placed in a $4 \mathrm{~L}$ beaker containing $3 \mathrm{~L}$ of water at a temperature of $25 \pm 1^{\circ} \mathrm{C}$. Tracking was performed with EthoVision for a period of $5 \mathrm{~min}$. The experiment was performed under red light. Animals were analyzed for the amount of time-spent mobile vs immobile.

Tail suspension test (TST). For the tail suspension test, which measures despair-like behaviors, each mouse was suspended with a long adhesive tape (taped $2 \mathrm{~cm}$ from tip of tail, $\sim 20 \mathrm{~cm}$ above from a shelf bottom). Its tail was passed through a small paper cylinder (width $1.5 \mathrm{~cm}$, height $2 \mathrm{~cm}$ ) before suspension to prevent climbing. Tracking of recorded video was performed with EthoVision for a period of $5 \mathrm{~min}$. The experiment was 
a

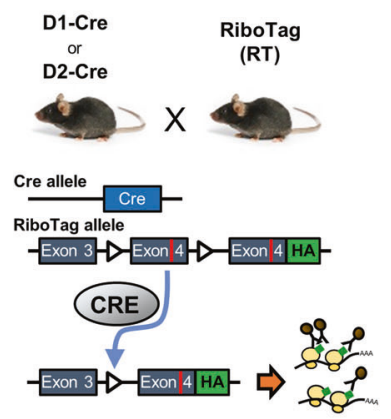

b
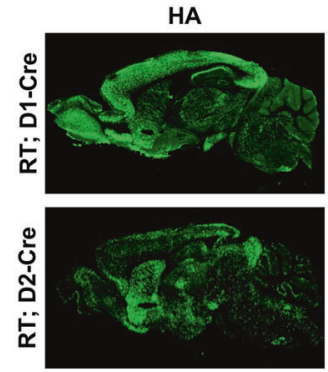
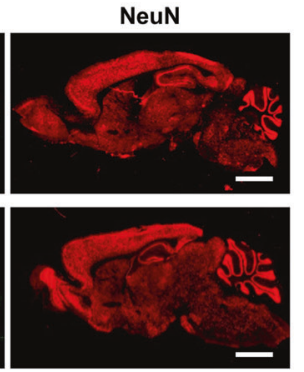

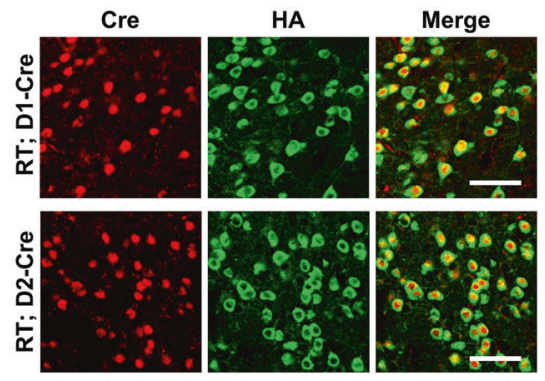

d
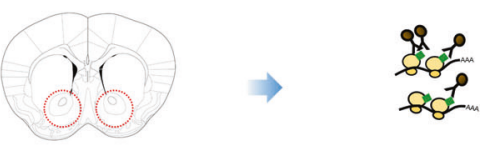

RiboTag IP

(D1 \& D2 cells)

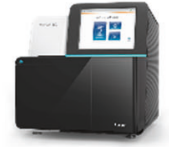

RNAseq

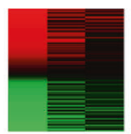

Analysis

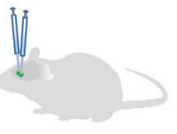

Validation f

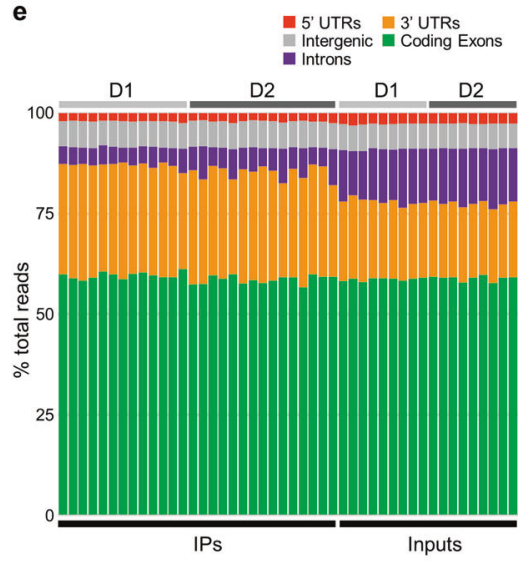

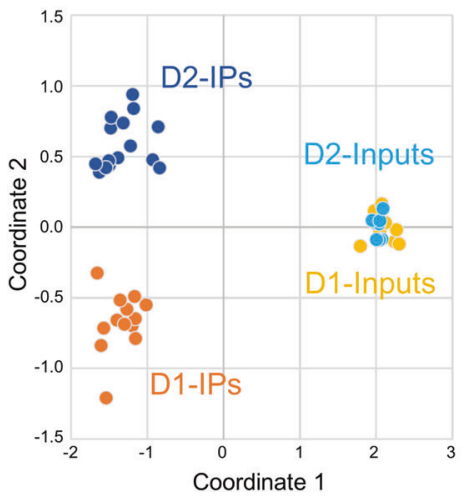

g

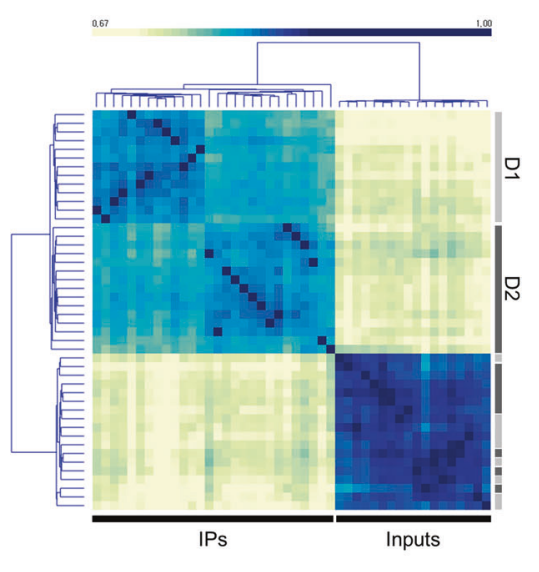

Fig. 1 MSN subtype specific transcriptomics in the NAc. a Schematic diagram of RT; D1-Cre and RT; D2-Cre mice generation and purification of MSN-subtype specific transcripts. The exogenous exon 4-HA cassette is expressed when the floxed exon 4 is excised in Cre-expressing cells. The HA-tagged polyribosome allows cell-type specific mRNA preparation from D1-MSNs (RT; D1-Cre) or D2-MSNs (RT; D2-Cre). b, c Validation of RiboTag mice with immunohistochemistry across brain regions (sagittal; scale bar, $2 \mathrm{~mm}$ ) (b) and in the NAc (coronal; scale bar, $50 \mu \mathrm{m}$ ) sections (c). d Schematic diagram of experimental design. e Read mapping composition of each RNA-seq sample on the mouse genome, mm10 (\% average for coding regions: IPs, 59.2\% vs inputs, 58.8\%; for intronic regions: IPs, 5.5\% vs inputs, $13.4 \%$ ). f Clustering of RNA-seq samples of RT; D1- and D2-Cre in a MDS plot. g Sample correlation heatmap were plotted with clustering (The dendrogram annotations on the right side axis: D1, light gray; D2, dark gray). For experiments in (e-g) D1-IPs, $n=13$; D2-IPs, $n=15$; D1-inputs, $n=9$; D2-inputs, $n=9$.

performed under white light conditions. Animals were analyzed for the amount of time-spent mobile vs immobile.

Sucrose preference. To assess natural reward using the sucrose preference test, $50 \mathrm{ml}$ tubes containing stoppers fitted with ballpoint sipper tubes were filled with solutions containing either $1 \%$ sucrose diluted in drinking water, or drinking water alone. The weights of solutions were recorded, and the position of the tubes was interchanged daily to eliminate side bias. Sucrose preference was calculated as a percentage of sucrose intake over total fluid volume consumed and averaged over 3 days of testing.

\section{Statistical analysis}

Statistical analyses of transcriptome data are described in the RNA-seq data analysis section. For all other analyses, unpaired or paired Student $t$ tests, one-way or two-way ANOVAs with Tukey's or Sidak's post-hoc test were performed to determine significance for conditions in which there were more than two groups or two factors. Statistical analysis was performed using Prism (version 8.3.0; GraphPad, San Diego, CA). To assess the homoscedasticity and normality of the distribution, the Shapiro-Wilk normality test was performed before parametric statistical analyses. We performed power analyses based on previous data using Statmate
(Graphpad). A sample size of 7 in each group of social defeat experiments has a $95 \%$ power to detect a difference between means of 42.03 (SI ratio) with a significance level (alpha) of 0.05 (two-tailed). All values included in the figure legends represent means \pm s.e.m.

\section{RESULTS}

\section{D1- and D2-MSNs express distinct transcriptomes}

D1- and D2-MSNs in the NAc have distinct and opposite roles in despair-like behavior [10-12], which suggest differential regulation of gene expression in each subtype. To evaluate the transcriptomic profile of MSNs, we generated mouse lines targeting D1- or D2-MSNs by crossing RT mice [8] with D1- or D2-Cre transgenic lines (RT; D1-Cre and RT; D2-Cre). This allows cell-type specific isolation of transcripts from D1- or D2-MSNs in the NAC (Fig. 1a). HA-tagged ribosomes express in a Credependent manner and thus only a subset of neurons, expressing D1- or D2-receptors are positive for HA (Fig. 1b, c, Supplementary Fig. 1). To analyze D1 and D2 transcriptomes in an unbiased manner, RNA sequencing (RNA-seq) experiments were performed 
7320

as depicted in Fig. 1d. On average, $92 \%$ of $19.3 \mathrm{M}$ reads from each sample was aligned with a high Q30 value (89\% bases above Q30), validating high quality of sequencing from RT samples (Supplementary Table. 1) in accordance with relatively uniform distributions of unnormalized counts (Supplementary Fig. 2a). IP samples are enriched with mature mRNA which has less intronic regions, whereas transcripts from input samples have more immature, premRNAs containing introns (Fig. 1e), as reported previously [9, 18]. Normalized gene expression profiles were obtained and plotted for each sample in a multidimensional scaling (MDS) plot, which showed distinct clustering of transcriptional profiles for each MSN subtype in IP samples (Euclidean distance: 1.258), but not in inputs which contain mixed population of cell-types in the NAC (Euclidean distance: 0.062) (Fig. 1f). In addition, unsupervised sample correlation mapping shows separate clustering of D1 and D2 groups in IP samples but not in input samples (Fig. 1g). Indeed, pairwise comparisons between independent biological replicates in D1 or D2 groups show high correlation values: avg. correlation in $\mathrm{D} 1, R=0.994$; in D2, $R=0.993$ (Supplementary Fig. 2b, c). The results from the MDS and correlation mapping are indicative of successful isolation and sequencing of distinct MSN subtypes in the NAc.

Next, to validate that NAc MSNs were isolated into distinct neuronal subtypes, we analyzed expression of well-characterized D1- and D2-MSN gene markers [19-22] and found enrichment of marker genes in each subtype as depicted in the heatmap and scatter plot (IP samples of RT; D1-Cre: Drd1 (2.4x), Chrm4 (1.8X), Tac1 (1.7x), and Pdyn (5.1X); RT;D2-Cre: Drd2 (3.6x), Gpr6 (2.8X), Adora2a (2.8X), and Penk (2.7x)) (Fig. 2a, b). MSN (Darpp-32) and neuron (NeuN) specific genes are enriched in both D1 and D2 samples at similar levels, whereas a glia-specific marker, Gfap, is depleted from IP samples by $90 \%$ relative to inputs, demonstrating enrichment of neuron specific genes. Visualized read coverages for selected marker genes in D1 and D2 samples by Integrated Genomics Viewer (IGV) [23] clearly illustrates celltype specific gene enrichment (Fig. 2c, d), whereas nonneuronal markers are depleted in the IP samples (Supplementary Fig. 3). These results were further validated by quantitative PCR (qPCR) using independent biological samples (Fig. 2e), which validate the reproducibility of RNA-seq quantification using RT and demonstrate our ability to efficiently segregate D1- and D2-MSNs with minimal cross-contamination.

We determined that there were 482 DEGs between D1- and D2MSNs ([Fold change (FC) $>1.5$, adjusted $p$ value $<0.05$ ), GO analysis of the 482 DEGs revealed that gene modules involving G-protein coupled receptors, synaptic transmission and neuropeptide related gene sets were enriched (Fig. 2f, Supplementary Table. 2). We analyzed the reproducibility of our results by comparing our dataset with a recently published report using the same RT method in the NAc [24]. To minimize inter-data variations between our results (Kim et al.) and Kronman's [24], we analyzed and compared both datasets using the same analysis workflow in Galaxy [25]. We found approximately a $40 \%$ overlap in gene expression in both D1 and D2 (top 100) genes between Kronman et al. [24] and our dataset (Fig. 2g), which demonstrates concordance between the two studies.

As the opposing roles of D1- and D2-MSNs are important to understand the neurobiology of depression [10-12], we further analyzed D1 and D2 transcriptomes in order to detect novel MSN subtype specific genes. Toward this end, we compared our data with two different MSN transcriptome datasets; one from RNA-seq analysis using RT [24] and the other a BAC-TRAP based microarray experiment [21] (Supplementary Fig. 4a). Overall, nearly a quarter of genes (D1, 93/339: 27.4\%; D2, 36/143: 25.2\%) from our datasets are shared with both studies, including all D1 and D2 marker genes tested in Fig. 2. More importantly, there are other, uniquely detected genes from both D1- (246 genes) and D2-comparisons (107 genes) in our datasets (Supplementary Fig. 4a). Selected genes from the lists, sorted by fold differences and adjusted $p$ values, show strong correlation between RNA-seq and qPCR (Pearson's correlation, $R=0.966$ ), which were further validated with in situ hybridization for Oxtr, Kcnj3, Ntrk1, and Tacr1 (Supplementary Fig. 4b-e). These results establish the validity of RT to detect novel cell-type specific mRNA transcripts and provide information on expression patterns at single cell levels, implicating transcriptional variability within subpopulations of each MSN subtype (Supplementary Fig. 4e).

\section{D1 transcriptome correlates with susceptibility}

To determine the transcriptional networks differentially regulated by stress in D1- and D2-MSNs, we applied CSDS to RT lines (RT; D1 Cre and RT; D2 Cre) for 10 days and their susceptibility or resilience to chronic stress was assessed using the SI test $[14,15]$ (Fig. 3a, b). Susceptible groups in both RT lines exhibit significant decreases of SI ratio (Fig. 3c; RT; D1-Cre, $F_{(2,23)}=100.9, p<0.001$ (4.09E-12); RT; D2-Cre, $F_{(2,27)}=54.63, p<0.001$ (3.23E-10)), while their locomotor activity levels were not changed (Supplementary Fig. 5). For transcriptomic analysis, we combined tissue from two mice which show similar levels of SI ratio, and performed RT immunoprecipitation followed by RNA-seq. D1 and D2 transcriptomes from each group (control, Res and Sus) were analyzed to detect DEGs for Sus vs Con, Res vs Con, or Sus vs Res with DeSeq2 ( $p$ value < 0.05 , vs control or Res) (Fig. 3d). Interestingly, within the D1 dataset, Sus group (vs Con, 923 DEGs) show approximately three times more DEGs than Res group (vs Con, 261 DEGs), whereas in the D2 dataset both Sus (vs Con, 442 DEGs) and Res group (vs Con, 403 DEGs) show similar number of DEGs (Fig. 3d, Supplementary Tables 3-8). Indeed, the third DEG set, Sus vs Res, in D1 shows 737 genes were differentially regulated, but the number is only 190 in D2, which indicates that Sus and Res transcriptome profiles are very different in D1, and relatively similar in D2. In addition, we compared the three different DEG lists for each cell-type in venn diagrams and found more genes are shared between Sus (vs Con) and Sus (vs Res) in D1, whereas, in D2, Sus (vs Con) and Res (vs Con) show more overlapped genes than Sus (vs Con) and Sus (vs Res) (Supplementary Fig. 6a, c). We hypothesized that genes showing distinct transcriptional profiles between Sus and Res are important in defining the Sus phenotype. Therefore, we created parallel plot heatmaps for Sus (vs Con) DEGs and compared Sus and Res profiles in each cell-type (Fig. 3e, Supplementary Fig. 6b, d). Broad patterns of transcriptional profiles show similar patterns in the D2 plot, and rather distinct in the D1 (Fig. 3e). For the D1 Sus DEGs (923 genes), only 73 genes (7.9\%) exhibited significant changes in the same direction $(p<0.05)$ in the D1 Res, whereas in the D2 Sus DEGs (442 genes), more genes (85 genes, 19.2\%) were changed also in D2 Res $(p<$ $0.05)$. Taken together, in terms of gene numbers and contents of the DEG lists, the D1 transcriptome, but not D2 transcriptome, shows distinct DEG profiles for Sus and Res (Pearson's correlation in D1, $R=0.759$; in $\mathrm{D} 2, R=0.849$ ) (Fig. 3e).

We hypothesized that genes showing opposite expression patterns between Sus and Res in D1 may play a role in the Sus phenotype. To identify the genes, we created a scatter plot from log2 fold difference data of D1 Sus (vs Con) and D1 Res (vs Con) (Fig. 3f). We found a total of 102 genes regulated in opposite directions in Sus and Res groups in the D1 dataset, whereas only 13 genes, mostly with marginal changes, were found in the D2 (Fig. 3f, Supplementary Fig. 6e, f). To determine their potential contribution to behavioral pathology, we performed GO analysis for D1 genes (102 genes) which revealed terms associated with neuronal function including synapse $(p=3.18 \mathrm{E}-08)$, dopaminergic- $(p=7.07 \mathrm{E}-04)$ and GABAergic-synaptic transmissions ( $p=$ 1.37E-03) (Supplementary Fig. 6h). Moreover, when compared to a curated gene set for depressive disorder (Harmonizome [26]), $\sim 40 \%$ of genes overlapped with the gene set for depressive disorders (Supplementary Fig. 6g), suggesting that the 102 
a

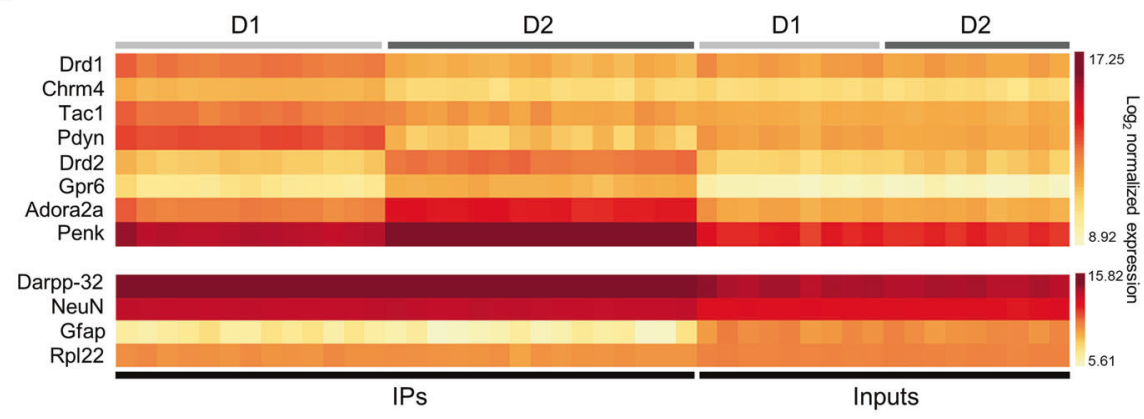

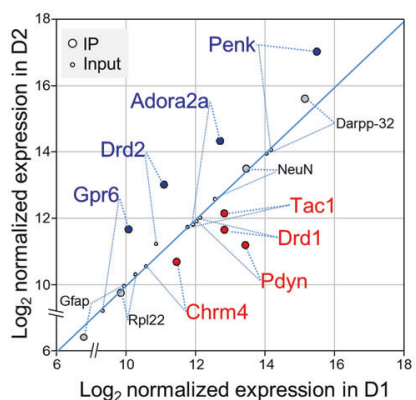

$\log _{2}$ normalized expression in D1
C

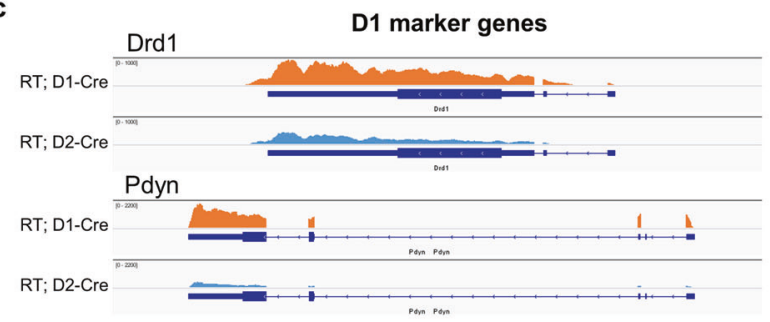

d

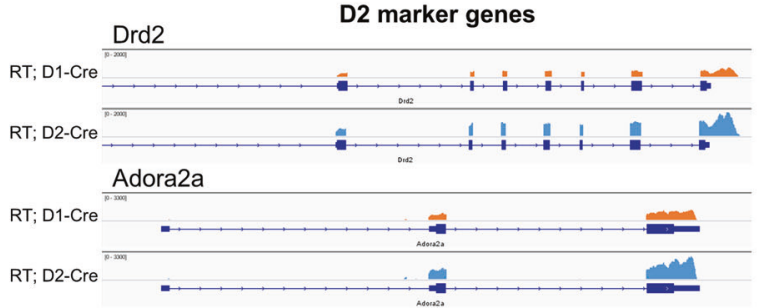

f
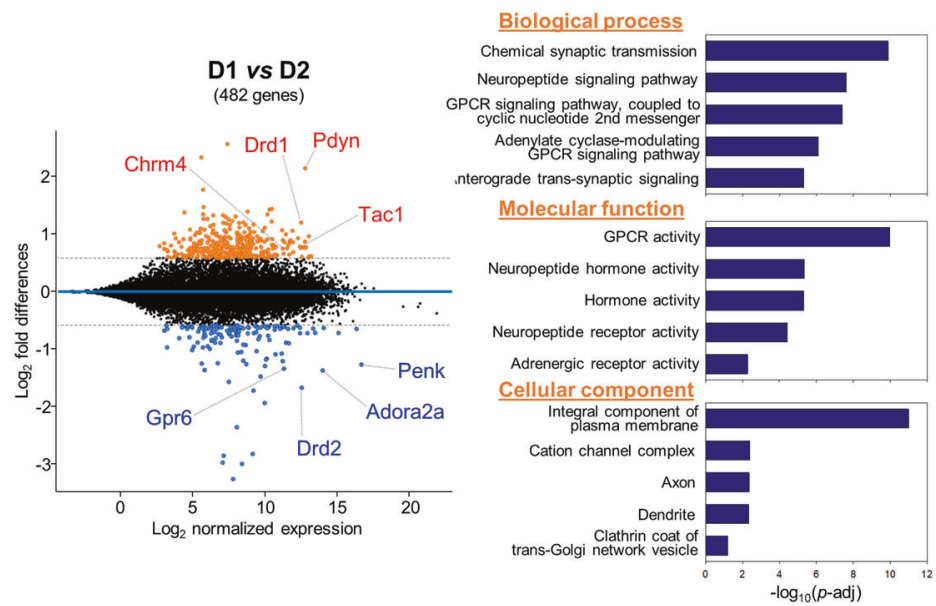

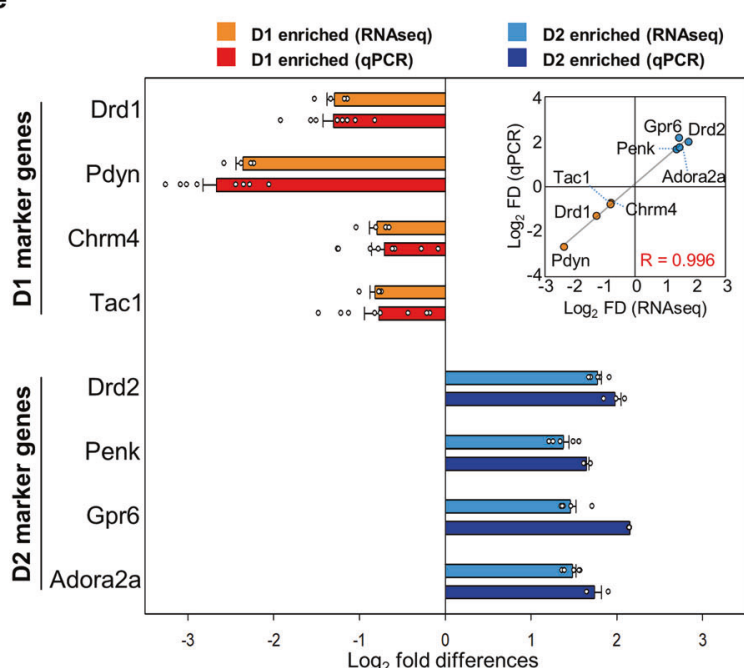

g

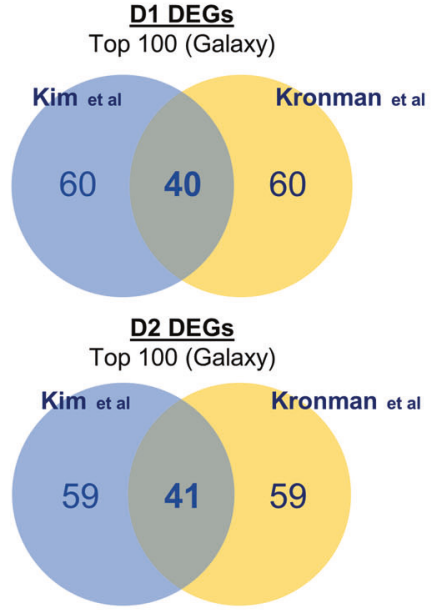

Fig. 2 Validation of MSN subtype specific transcriptomics with D1- or D2-MSN specific marker genes. a, b Heatmap (a) and scatter plot (b) visualization of normalized expressions for MSN-subtype specific marker genes (D1-IPs, $n=13$; D2-IPs, $n=15$; D1-inputs, $n=9$; D2-inputs, $n=$ 9). c, d Representative aligned reads on selected D1- (Drd1 and Pdyn) (c) or D2-marker genes (Drd2 and Adora2a) (d) were visualized and compared by IGV. e Fold differences of D1- or D2-marker gene expressions from RNA-seq (D1 enriched, $n=4$; D2 enriched, $n=5$ ) were plotted with independent qPCR validation data (D1 enriched, $n=8$; D2 enriched, $n=3$ ) in bar chart (e; mean \pm s.e.m.) and scatter plot (inset; Pearson's correlation, $R=0.9961$ ). f MA plot of 482 DEGs between D1 control and D2 control comparison (D1 enriched (orange dots), 339 genes; D2 enriched (blue dots), 143 genes; [Fold change $(\mathrm{FC})$ ] > 1.5, adjusted $p$ value $<0.05)$. Gene ontology (GO) analysis of the $482 \mathrm{DEGs}$ from the control group comparison. $g$ Comparisons between top 100 D1- or D2-enriched gene lists from Kim et al. and Kronman et al. by the same RNA-seq analysis workflow in Galaxy.

genes contain potential target genes functionally associated with susceptibility.

From the D1 Sus gene list, we focused on Shisa6 which showed increased expression in Sus, and decreased in Res, these changes were the largest among the 102 genes (Fig. 3f). Furthermore, from
D1 vs D2 comparisons (Supplementary Fig. 7, Supplementary Table. 2, 9, 10), we also found Shisa6 in the D1 Sus DEG list, which is associated with highly relevant GO terms such as MDD, bipolar disorder, and anxiety in a GWAS database (Supplementary Fig. 7d). These data suggest that Shisa6 may function as a potential pro- 

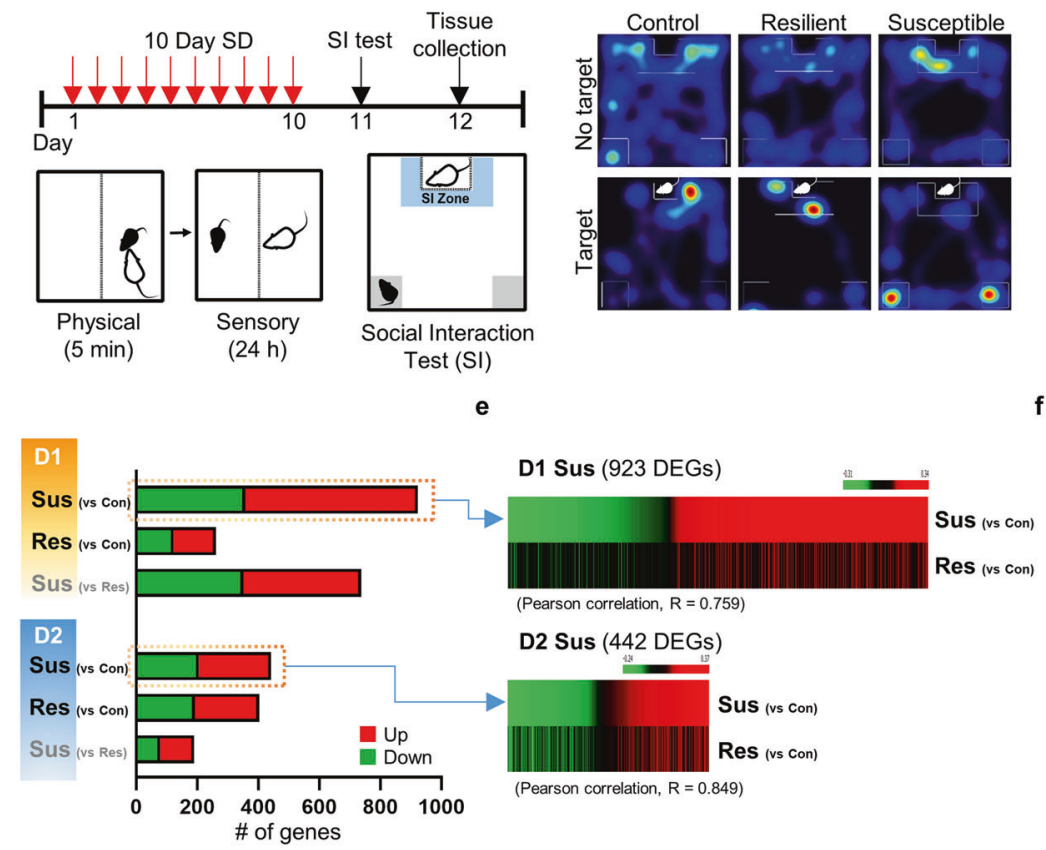

g

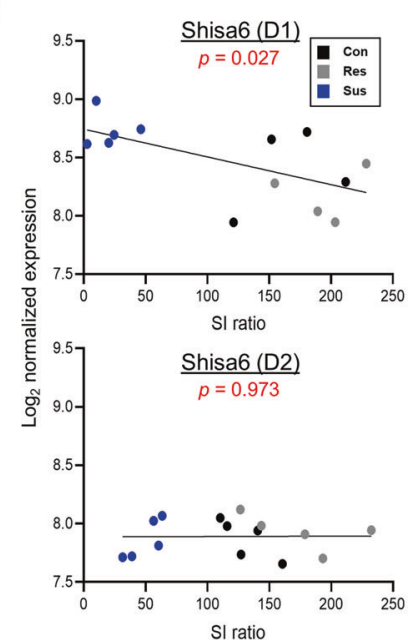

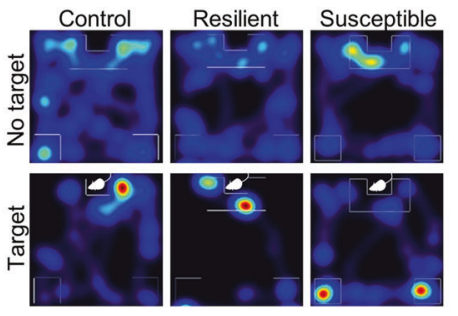

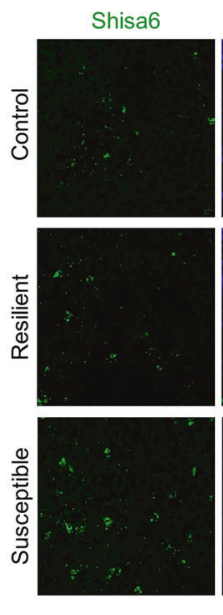

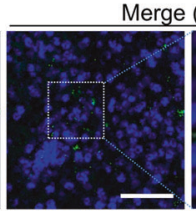
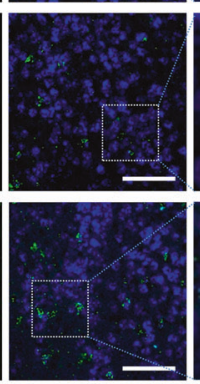

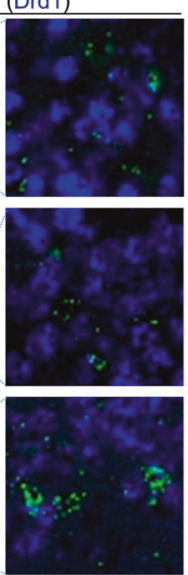

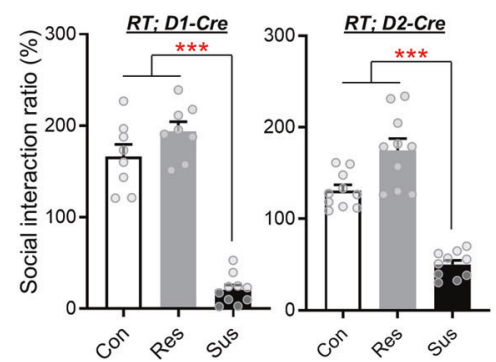

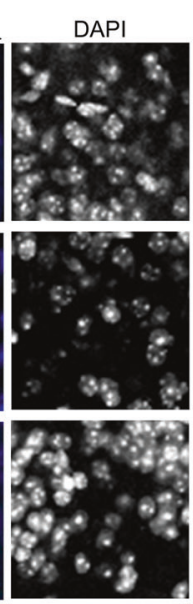

i

Fig. 3 Stress group-specific changes of gene expression in D1- and D2-MSNs. a, b Schematics of chronic social defeat stress (CSDS) (a) and representative heat maps of social interaction (SI) behavior (b). c SI ratio of RiboTag (RT); D1- or D2-Cre mice which were used for RNA-seq analysis (vs control or resilient, ${ }^{* * *} p<0.001 ; \mathrm{RT} ; \mathrm{D} 1-\mathrm{Cre}, F_{(2,23)}=100.9 ; \mathrm{RT} ; \mathrm{D} 2-\mathrm{Cre}, F_{(2,27)}=54.63$; calculated by one-way analysis of variance (ANOVA) with Tukey's post-hoc test; D1: Con, $n=8$; Res, $n=8$; Sus, $n=10$; D2: Con, $n=10$; Res, $n=10$; Sus, $n=10)$. d Numbers of DEGs from comparisons between stress groups are plotted (threshold, $p<0.05$; \# of DEGs for D1 comparisons: Sus vs Con, 923 (566 up; 357 down), Res vs Con, 261 (138 up; 123 down) and Sus vs Res, 737 (386 up; 351 down); for D2, Sus vs Con, 442 (237 up; 205 down); Res vs Con, 403 (211 up; 192 down) and Sus vs Res, 190 (111 up; 79 down)). e D1- and D2-susceptible DEGs (vs control, $p<0.05)$ are sorted by fold change (FC) values and compared to FC values of corresponding resilient DEGs (vs control, $p<0.05$ ). f A scatter plot of D1-susceptible DEGs with log2 fold difference values shows inversely modulated genes (filled circles, 102 genes) in susceptible and resilient groups. $\mathbf{g}$ Shisa6, one of the 102 genes, inversely correlates with SI ratio in D1, but not D2. $p$ values in linear regression. $\mathbf{h}-\mathbf{j}$ Validation of Shisa6 expression in the NAc of socially defeated mice using in situ hybridization (RNAscope; scale bar, $100 \mu \mathrm{m})(\mathbf{h})$. Average expression at single cell level (i) and \% colocalization $(\mathbf{j})$ of Shisa6 ${ }^{+}$cells in D1-MSNs $\left(\mathrm{Drd1}^{+}\right)$(mean \pm s.e.m.; ${ }^{*} p<0.05,{ }^{* *} p<0.001$; n.s. not significant; expression/cell, $F_{(2,53)}=4.705 ; \%$ colocalization, $F_{(2,53)}=8.507$; calculated by one-way ANOVA with Tukey's post-hoc test). For experiments in (d, g) D1 Con, $n=4$; D1 Res, $n=4$; D1 Sus, $n=5$; D2 Con, $n=5$; D2 Res, $n=5$; D2 Sus, $n=5$. For experiments in (i, j) Con, $n=16$; Res, $n=16 ;$ Sus, $n=24$ from 4 Con, 4 Res, and 6 Sus mice.

depressive gene in D1-MSNs. Shisa6 was recently identified as a component of AMPA receptors (AMPAR), which is expressed in various brain regions (Supplementary Fig. 8), and sustains AMPAR currents by preventing desensitization [27]. Recently, Shisa6 was found in a proteome analysis of developing synapses [28] in addition to facilitating purkinje cell synaptic excitability and procedural memory [29]. Interestingly, Shisa9, which has opposite functions to Shisa6 at glutamatergic synapses [27], was identified as a highly associated gene for major depression in recently published large-scale GWAS studies [30, 31]. Here we show a strong inverse correlation between Shisa6 expression and SI solely in D1-MSNs $(p=0.027)$, but not in the D2-MSNs $(p=0.973)$ (Fig. $3 \mathrm{~g}$ ). We further validated the inverse correlation between Shisa6 expression in D1 and SI with in situ hybridization assay 
using an independent social defeat cohort, which showed higher expression of Shisa6 in D1-MSNs of Sus mice (Fig. 3h-j). Only the Sus group showed a significant increase of Shisa6 expression by $22 \%$ in each cell (Fig. 3i), which corresponds to RNA-seq data (Fig. $3 g$ ). Shisa6 ${ }^{+}$D1 cells in Sus mice (23.3\%) show similar \% colocalization with the Con (20.8\%), and only the Res group (15.9\%) exhibits significant decrease in colocalization (Fig. 3j), which implicates only small subsets of D1-MSNs express Shisa6, and the Shisa6 expression is controlled in the restricted D1 populations according to Sus or Res phenotype.

\section{Shisa6 functions in a circuit-specific manner}

We validated that Shisa6 shows increased expression in D1-MSNs of Sus mice via RNA-seq and in situ hybridization (Fig. 3g-j), which may suggest a pro-Sus role for Shisa6 in D1-MSNs. To investigate potential causal relations of Shisa6 expression and its pro-Sus effects, we designed a cell-type specific AAV vector to ectopically increase Shisa6 expression only in D1-MSNs and measure subsequent changes in neural activity and behavior (Fig. 4). The AAV-DIO-Shisa6-mCherry (Supplementary Fig. 9) or -mCherry only control was injected in the NAC of D1-Cre mice bilaterally and D1MSN specific expression was assessed with in situ hybridization (Fig. 4a, b). Localization of ectopically expressed Shisa6 protein was also tested with immunohistochemistry, which shows intense localization at the membrane and neural processes of infected D1MSNs (Supplementary Fig. 9b).

For the electrophysiological analysis, acute brain slices were prepared from Shisa6 or control AAV injected Ai6; D1-Cre mice and the AAV infected D1-MSNs (mCherry and ZsGreen 1 doublepositive) were recorded (Fig. 4c, Supplementary Fig. 10). The amplitude, but not frequency, of spontaneous excitatory postsynaptic currents (sEPSCs) was significantly increased in Shisa6-overexpressing D1-MSNs compared with controls (Fig. 4c). In addition, AMPA/NMDA ratio was increased in the Shisa6 overexpressing D1-MSNs (Supplementary Fig. 10b). In contrast, no significant alteration was found in the spontaneous inhibitory postsynaptic currents or action potentials evoked by current steps between control and Shisa6 overexpressing D1 neurons (Supplementary Fig. 10c, d). We next conducted morphological analysis on the recorded D1-MSNs using biocytin injection. No significant change in dendritic complexity (length and number of intersections) was observed between control and Shisa6 groups (Fig. 4d). Taken together, these results suggest that Shisa6 overexpression in D1-MSN induced augmented synaptic responses to glutamatergic inputs without significant alteration of dendritic structure.

Considering the role of Shisa6 in AMPA receptor function, the increased amplitude of sEPSC (Fig. 4c) is highly indicative of a functional coupling of Shisa6 and AMPAR currents at excitatory synapses. To examine localization of Shisa6 in functionally relevant glutamatergic synapses in D1-MSNs, we labeled synapses receiving inputs from two major excitatory afferent sources to the NAc, the medial prefrontal cortex (mPFC) and the ventral hippocampus (vHIP), with dual-eGRASP [32] (Fig. 4e). Two different AAVs, which express post-eGRASP or Shisa6 in a Cre-dependent manner, were injected together in the NAc of D1-Cre mice. We also injected two different pre-eGRASP AAVs, which express cyan- or yelloweGRASP, in the mPFC and vHIP, respectively. We found sparsely or densely labeled eGRASP positive synapses throughout the NAC region (Supplementary Fig. 11). Ectopically expressed Shisa6 is well colocalized with both excitatory synapses, receiving inputs from mPFC (cyan) and vHIP (yellow) (Fig. 4f), and these results strongly indicate a functional role for Shisa6 in glutamatergic synapses, which may be relevant to the Sus phenotype (Fig. 4c).

It is of interest to ascertain whether upstream neural inputs to D1-MSNs in the NAc mediate Shisa6 expression depending on Sus or Res status. Firing patterns of ventral tegmental area (VTA) dopamine neurons are important to determine susceptibility or resilience to social stress $[16,33,34]$, therefore we evaluated whether activation of the VTA to NAc circuit can mediate Shisa6 expression in D1-MSNs. Phasic activation of VTA to NAc circuit is a model known to exert pro-Sus effects in mice [33, 34], and is useful to assess pro-Sus genes in the NAc $[35,36]$. To activate the VTA to NAc pathway, we specifically labeled the circuit with ChR2 (or EYFP as control) and stimulated the fiber ends in the NAc to ensure region specific activation (Fig. 4g, Supplementary Fig. 12). After 10 day-chronic activation of the circuit, we resolved cell-type specific expression of Shisa6 with RT immunoprecipitation for both D1- and D2-MSNs. Interestingly, Shisa6 showed approximately a $50 \%$ increase in D1-MSNs, whereas there was no change of Shisa6 expression in D2-MSNs (Fig. 4h). These results suggest VTA neurons, which project to D1 are direct upstream modulators of Shisa6 expression.

\section{Shisa6 modulates behavioral susceptibility}

To investigate whether Shisa6 can directly modify depression- and anxiety-like behaviors in vivo, we overexpressed or knocked-down the Shisa6 gene in D1-MSNs and performed several behavioral assays. First, we examined baseline behavioral changes upon Shisa6 overexpression in D1-MSNs of male and female mice (Supplementary Fig. 13). Baseline behavioral assessments showed increased anxiety-like behaviors (decreased center time in openfield test; *, $p=0.011$ ) and anhedonia (decreased sucrose preference; ${ }^{*}, p=0.040$ ) when Shisa6 is overexpressed in D1MSNs of male mice (Supplementary Fig. 13a-i). In the same baseline condition, female mice showed a similar trend of anhedonia (decreased sucrose preference; \#, $p=0.071$ ) after Shisa6 overexpression in D1-MSNs, while other behaviors showed decreasing trends of anxiety (increased open arm time in elevated-plus maze; \#, $p=0.054$ ) and despair-like behaviors (decreased immobile time; \#, $p=0.073$ ) (Supplementary Fig. $13 \mathrm{j}-\mathrm{q}$ ). With the male mice which overexpress Shisa6 in D1MSNs, we conducted an additional acute social defeat experiment to assess the effects of Shisa6 increase prior to social stress exposure (Supplementary Fig. 13a). Interestingly, after acute social defeat stress (sub-maximal social defeat), the Shisa6 overexpressed male mice exhibited no significant decrease of $\mathrm{SI}$ (Supplementary Fig. 13i). To determine if Shisa6 acts in a prodepressive manner in mice previously exposed to social stress, we selectively increased Shisa6 in D1-MSNs after CSDS (Fig. 4i). Overexpression of Shisa6 resulted in a decreased trend for SI (Fig. 4j; left panel, decreased by $35.6 \%$ (vs DIO-mCherry control), ${ }^{\#,}$ $p=0.082$; right panel, Shisa6 overexpression (Pre vs Post), n.s., $p=$ $0.101)$. Next, to assess if blocking Shisa6 could rescue deficits in SI post CSDS, we selectively knock-down Shisa6 by overexpressing shRNAs targeting Shisa6 mRNA in D1-MSNs (Supplementary Fig. 9d-f). We found that SI was significantly increased in the Shisa6 knock-down group (Fig. 4k; left panel, increased by $56.8 \%$ (vs shRNA scrambled control), ${ }^{*}, p=0.038$; right panel, Shisa6 knock-down (Pre vs Post), $p=0.015$ ). These results demonstrate that Shisa6 knock-down in D1-MSNs can directly rescue depression-like behaviors, by increasing SI.

\section{DISCUSSION}

Here we report the first cell-type specific transcriptomic analysis of socially defeated mice and identify novel DEGs in D1- and D2MSNs, that may contribute to the pathophysiology of depression. We revealed that distinct subsets of genes, directly related to neuronal function, were differentially expressed in D1- and D2MSNs of Sus mice. We identified more DEGs in the Sus group than Res in D1-MSNs, whereas similar numbers of DEGs were detected in both Sus and Res D2-MSNs. Although, cell-type specific genes linked to depression have been identified in chronic stress models $[10,37-41]$, transcriptome-wide stress-induced changes in specific neurons in the NAC have yet to be reported. 

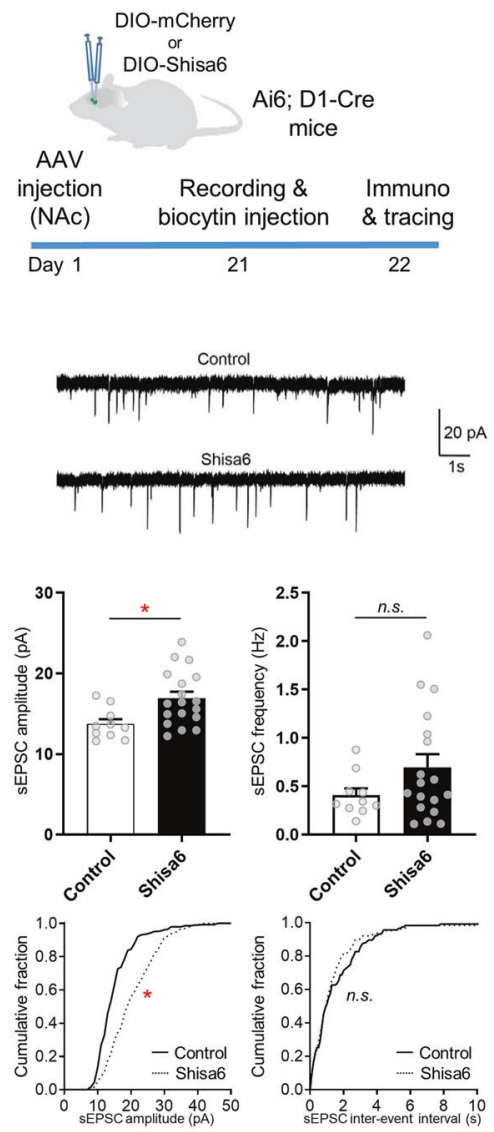

g
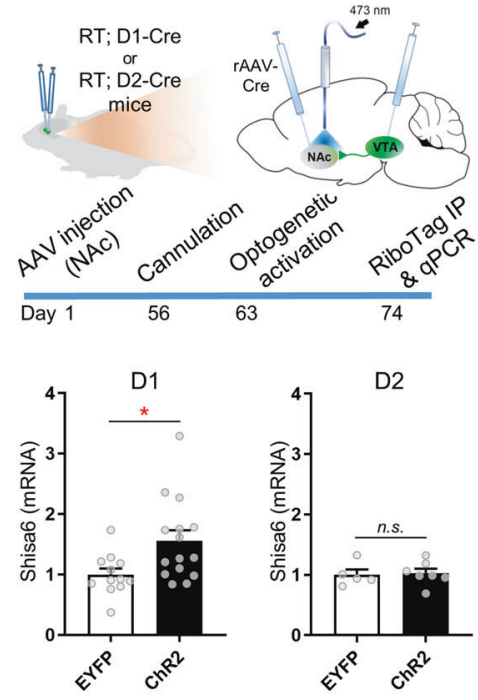

b

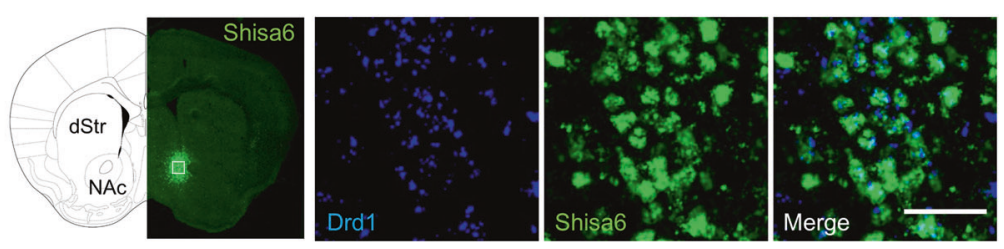

d

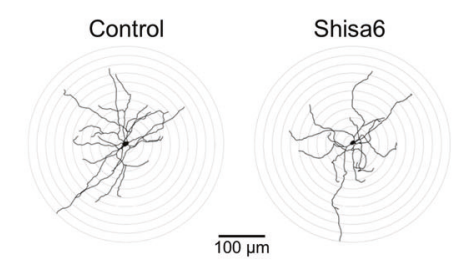

e

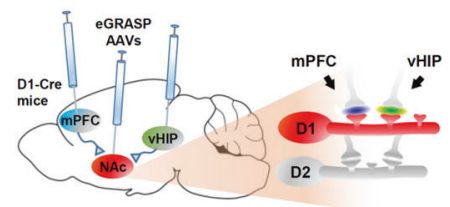

f

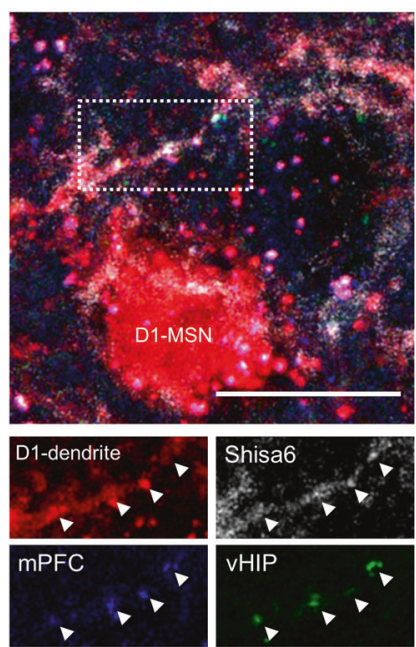

i
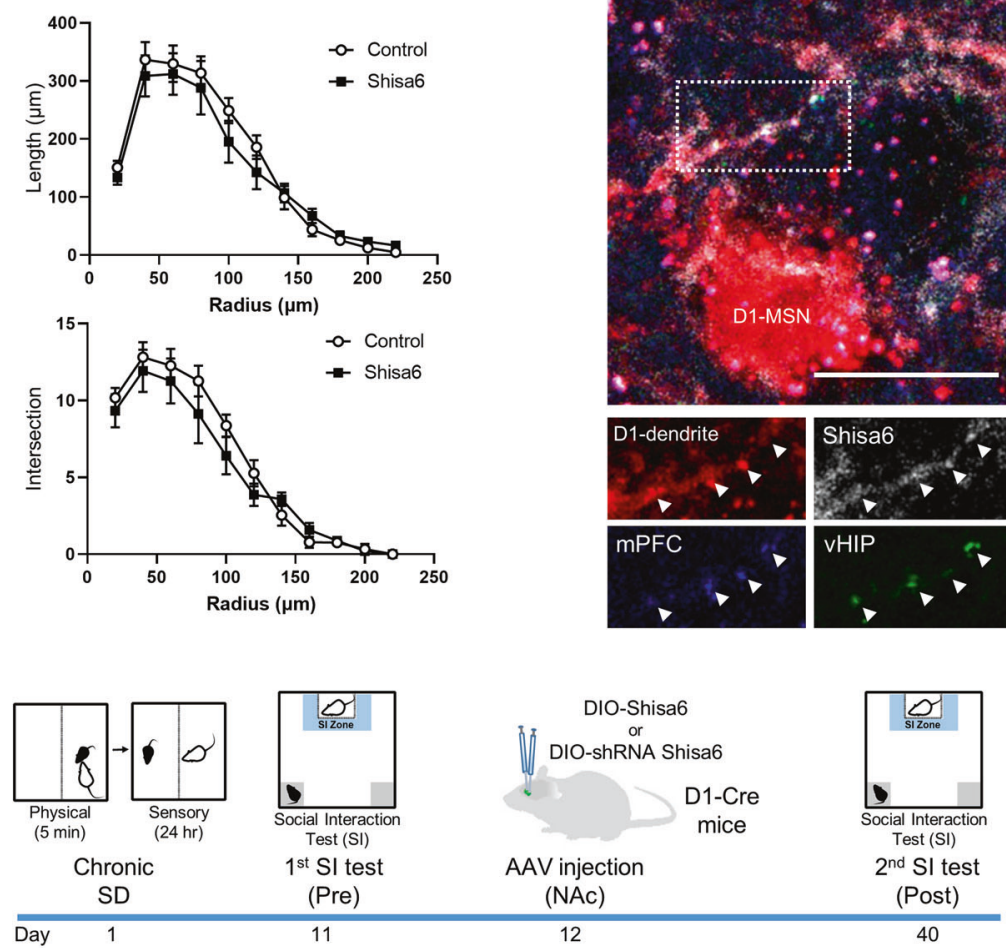

j

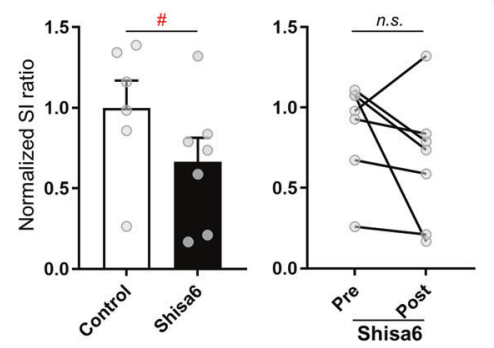

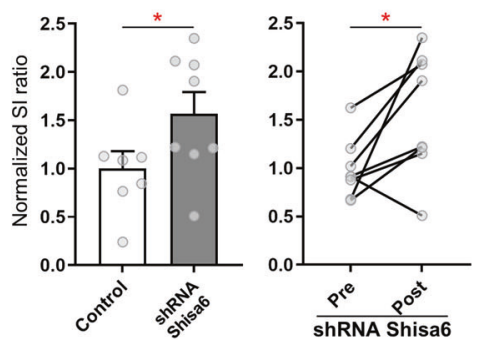

Gene expression patterns in Sus and Res are distinct in D1, as compared to D2, which suggest D1-MSNs mediate Sus phenotypes such as social avoidance and anhedonia, while D2-MSNs act on common stress pathways in both Sus and Res mice. We further analyzed the Sus and Res genes by comparing the same stress groups between D1- and D2-MSNs and found approximately half of the genes on the D1 lists show significant correlations to SI which highlights the importance of D1-MSNs in the regulation of the Sus phenotype (Supplementary Fig. 7). We found a group of Sus specific genes in D1-MSNs showing opposite direction of regulation when compared to Res mice (102 genes), and amongst the genes, we identified Shisa6 as a potential candidate for the regulation of D1-MSNs in mice exposed to social stress. Shisa6 expression levels are significantly increased in a subset of D1MSNs and their levels are inversely correlated with the SI ratio. Interestingly, although cells in Res express Shisa6 comparably to Sus, they are not highly colocalized with D1-MSNs, which suggest Shisa6 in D2-MSNs or non-MSN populations act differently in Res mice. Recent single-cell RNA-seq analyses reveal subpopulations for each MSN subtypes [22, 42-44] and future studies should 
Fig. 4 Functional validation of Shisa6 related to depression phenotypes. a Schematics of experimental plan of electrophysiological and morphological analyses. b Validation of D1-MSN specific viral expression of Shisa6 (in situ hybridization; scale bar, $50 \mu \mathrm{m}$ ). c Representative sEPSC traces, bar graphs (mean \pm s.e.m.) and cumulative distribution of sEPSC amplitude and frequency in D1-MSN from control vs Shisa6 comparisons (amplitude: control, $14.4 \pm 0.8$ pA; Shisa6, $16.9 \pm 0.8$ pA; frequency: control, $0.41 \pm 0.07 \mathrm{~Hz}$; Shisa6: $0.69 \pm 0.13 \mathrm{~Hz}$; control, $n=10$; Shisa6, $n=18$ ). d Morphological analysis of biocytin-injected control or Shisa6-overexpressing D1-MSNs (control, $n=11 ;$ Shisa6, $n=15$ ). e, $\mathbf{f}$ Afferent input specific excitatory synapse labeling (eGRASP) and Shisa6 colabeling in D1-MSNs. Schematics of D1-MSN specific eGRASP in the NAc (e) and colocalization of Shisa6 at mPFC and vHIP afferent synapses (f; arrowhead: mPFC or vHIP eGRASP, scale bar: $20 \mu \mathrm{m}$ ). $\mathbf{g}$, $\mathbf{h}$ Chronic optogenetic activation of VTA to NAc circuits and MSN subtype specific Shisa6 gene expression analysis. $\mathbf{g}$ Schematics of optogenetic experiments. VTA to NAc circuit of RT; D1-Cre or RT; D2-Cre mice was labeled with retrograde Cre-AAV (rAAV2-Cre in the NAc) and Cre-dependent ChR2 or EYFP AAVs (in the VTA) and then implanted with optic-cannula in the NAc. Mice received optogenetic activation $(20 \mathrm{~Hz}$, phasic $473 \mathrm{~nm}$, for $5 \mathrm{~min} /$ day) for 10 days. h RiboTag IP and qPCR analysis of Shisa6 gene expression in D1- or D2-MSNs after 10 day optogenetic activation (D1: EYFP, $n=12$; ChR2, $n=15$; D2: EYFP, $n=5$; ChR2, $n=7$ ). i-k Behavioral analysis of D1-MSN specific Shisa6 modulation in socially defeated mice. i Schematics of behavioral experiments and timeline. $\mathbf{j}$, $\mathbf{k}$ Assessments of social interaction changes after Shisa6 overexpression (j; control (DIO-mCherry), $n=6$; Shisa6, $n=7$ ) or knock-down (k; control (shRNA scrambled), $n=7$; shRNA Shisa6, $n=8$ ) in D1-MSNs. Values were normalized to the control. Graphs are represented as mean \pm s.e.m. $\left(^{*} p<0.05 ;{ }^{\#} p<0.09 ; n . s\right.$. not significant; $t$-test, two-tailed (c, h), one-tailed (j, k); two-way ANOVA with Sidak's post-hoc test (d)).

examine regionally defined subpopulation of MSNs (e.g., NAc shell vs core regions) $[22,45]$ or conduct circuit specific single cell analysis [46].

Shisa family members are single transmembrane adaptor proteins that regulate signaling pathways [47, 48] including neuronal transmission by altering glutamatergic or GABAergicsynaptic transmission through direct interactions with AMPAR [27] (Shisa6 and Shisa9) or GABA $A_{A} R$ [49] (Shisa7). Increased expression of Shisa6 is expected to preserve synaptic AMPAR and prevent desensitization in D1-MSNs [27], which increases overall AMPAR current. Indeed, D1-MSNs in which Shisa6 is overexpressed show increased SEPSC amplitudes without changing dendritic arborization. Moreover, the increased EPSC amplitudes are exclusively driven by AMPAR-EPSC which validate Shisa6 mediated facilitation of AMPAR function. This is consistent with the observation that Shisa6 protein is localized to glutamatergic synapses, from two major afferent sources, mPFC and vHIP. In our in situ hybridization experiment, $20 \%$ of D1-MSNs express detectable amounts of Shisa6 mRNAs, and it is of interest whether the Shisa6 ${ }^{+}$D1-MSN subpopulation has specific neural connections with other brain regions relevant to depressive-like phenotypes including VTA, $\mathrm{mPFC}$, and vHIP. Interestingly, Shisa9 was recently identified as a gene related to major depression by large-scale GWAS [30, 31] and is known to counteract Shisa6 [27]. We observed that Shisa9 showed a decreased trend of expression in D1-MSNs of Sus mice (Supplementary Fig. 8). Despite the fact that Shisa6 expression in D1-MSNs was correlated with SI after CSDS, Shisa6 in other celltypes might contribute to circuit level modulation of depressivelike phenotypes in the NAc.

Shisa6 expression is regulated in a cell-type specific manner following CSDS and optogenetic experiments suggest that hyperactivity of VTA dopamine neurons projecting to the NAC is a putative upstream signal mediating Shisa6 expression in D1MSNs but not D2-MSNs of Sus mice. Interestingly, optogenetic activation of the VTA- NAc circuit is sufficient to alter Shisa6 expression, but doesn't alter SI behaviors. This result suggests that non-context dependent activation of dopamine neurons is insufficient to recruit glutamatergic inputs from the MPFC and/ or vHIP to induce depressive-like phenotypes, which is supported by previous research [34, 35]. However, in stress conditions, Shisa6 may be localized to specific synapses which receive glutamatergic inputs relevant to depressive phenotypes. In this regard, it is noteworthy that vHIP-NAc glutamatergic circuit delivers aversive information only to D1-MSN, but not D2, and increased synaptic strength of excitatory synapses directly increased depression-like behaviors, concurrent with increased AMPAR transmission [50]. These results are well correlated with our data showing D1-MSN specific increase of Shisa6 and corresponding enhancement of excitability and depressive-like behaviors following CSDS. In the future, exploring the Shisa6 ${ }^{+}$D1-MSN population and their connectivity may provide valuable insight on mechanisms of depression.

Our data showing an increase in Shisa6 in Sus mice is consistent with the hypothesis that increased excitability of the NAc contributes to stress vulnerability. For example, Vialou et al. showed that, in Sus mice and humans that experienced MDD, there was increased AMPA receptor function (i.e., higher GluR1: GluR2 ratio) in the NAc [51]. Similarly, the decrease in Shisa6 in Res animals suggests that decreased glutamatergic function may be protective against impairing effects of chronic stress, which is consistent with studies demonstrating that decreased AMPA activity enhances reward-related behaviors [52, 53]. However, in contrast to our finding, it should be noted that other studies with different stress models [54-56] show a decrease in D1-MSN AMPA mediated excitation after stress. These conflicting results highlight the complex nature of NAC circuit regulation in depression.

In chronic stress conditions, excitatory transmission and intrinsic excitability are differentially regulated between D1- and D2-MSNs $[50,54,57]$. For example, D1-MSNs show increased intrinsic excitability in Sus mice after CSDS $[51,57,58]$, which corresponds to the effects of Shisa6 increase in Sus mice. This result might also be reflective of stress-induced reorganization of afferent circuits to D1-MSNs to selectively strengthen aversive afferent inputs, while at the same time decreasing connections from mPFC to NAc which promotes resiliency $[59,60]$. A recent study shows that $\mathrm{vHIP}$ sends projections to the NAc and the circuit directly mediates SI behaviors [61]. More importantly, the vHIP-NAc glutamatergic circuit delivers aversive information only to D1-MSN, but not D2, and increased synaptic strength of the excitatory synapses directly increased depression-like behaviors [50]. These findings are wellaligned with D1-MSN specific increase of Shisa6 and corresponding strengthening of AMPA currents at excitatory synapses including VHIP afferent synapses. Indeed, we show that downregulation of Shisa6 in D1-MSNs reversed social avoidance behavior in socially defeated male mice, which validates causal relations between Shisa6 and depressive phenotypes. On the other hand, overexpression of Shisa6 prior or post to social defeat only exerted marginal effects on SI behaviors. We speculate that the loss of Shisa6 function by knock-down might be more specific to the Shisa6 expressing- and stress-relevant D1-MSN subsets rather than the gain of function in a broad population of D1-MSNs by Shisa6 overexpression. Intriguingly, D1-MSN specific Shisa6 overexpression without social stress exposure induced anhedonia in both male and female mice, while some opposing behavioral responses were observed in anxiety- or depression-like behavioral assays, which implicate possible sex specific roles of Shisa6 in depression. Taken together, these data suggest that depressive phenotypes can be reversed by targeting Shisa6, as demonstrated in the Shisa6 knock-down experiment. 
Regulation of glutamate synapses is considered a potential target for antidepressants and our findings suggest that glutamatergic inputs to the NAc might be important in regulating depressive-like symptoms; given that we observe a selective increase of Shisa6, a bona fide AMPAR auxiliary subunit, in D1MSNs. The regulation of Shisa6, an AMPAR regulatory protein, may act as a potential therapeutic target for treating depression. Indeed, postmortem tissue of depression patients show an increase in radio-ligand binding of AMPA in anterior cingulate cortex [62], and preclinical stress models show an increase in expression of AMPA receptor subunits following acute [63] and chronic [63-65] stress, while other studies find a decrease in expression of specific AMPA receptor subunits following stress [66-69]. Importantly, AMPA receptor antagonist NBQX infused into the NAc increased SI time, demonstrating that inhibition of AMPA receptor function induces an antidepressant-like response [51]. These data are consistent with our observation that Shisa6 knockdown in D1-MSNs rescued social avoidance in socially defeated mice and show promising therapeutic applications of Shisa6 in modulating depression.

In summary, we found Shisa6 expression is increased in D1MSNs of Sus mice and increases excitability of neurons, which modulates anxiety- and depression-like behaviors in mice. Shisa6 is expressed in a cell-type and circuit-specific manner, and may directly alter the strength of pre-existing excitatory synapses related to aversive information flow. Taken together, Shisa6 may be a critical link of dopamine and glutamatergic systems in the reward circuitry, and a promising molecular target for intervention during depression pathogenesis.

\section{Reporting summary}

Further information on research design is available in the Nature Research Reporting Summary linked to this paper.

\section{DATA AVAILABILITY}

RNA-seq data have been deposited to the NCBI GEO under accession number GSE147640.

\section{REFERENCES}

1. Ledford H. Medical research: if depression were cancer. Nature. 2014;515:182-4.

2. Greenberg PE, Fournier AA, Sisitsky T, Pike CT, Kessler RC. The economic burden of adults with major depressive disorder in the United States (2005 and 2010). J Clin Psychiatry. 2015;76:155-62.

3. Fava M. Diagnosis and definition of treatment-resistant depression. Biol Psychiatry. 2003;53:649-59.

4. Holtzheimer PE, Mayberg HS. Stuck in a rut: rethinking depression and its treatment. Trends Neurosci. 2011;34:1-9.

5. McIntyre RS, Filteau MJ, Martin L, Patry S, Carvalho A, Cha DS, et al. Treatmentresistant depression: definitions, review of the evidence, and algorithmic approach. J Affect Disord. 2014;156:1-7.

6. Berton O, Nestler EJ. New approaches to antidepressant drug discovery: beyond monoamines. Nat Rev Neurosci. 2006;7:137-51.

7. Handley A, Schauer T, Ladurner AG, Margulies CE. Designing Cell-Type-Specific Genome-wide Experiments. Mol Cell. 2015;58:621-31.

8. Sanz E, Yang L, Su T, Morris DR, McKnight GS, Amieux PS. Cell-type-specific isolation of ribosome-associated mRNA from complex tissues. Proc Natl Acad Sci USA. 2009;106:13939-44.

9. Dougherty JD. The Expanding Toolkit of Translating Ribosome Affinity Purification. J Neurosci: Off J Soc Neurosci. 2017;37:12079-87.

10. Fox ME, Lobo MK. The molecular and cellular mechanisms of depression: a focus on reward circuitry. Mol Psychiatry. 2019;24:1798-815.

11. Francis TC, Lobo MK. Emerging Role for Nucleus Accumbens Medium Spiny Neuron Subtypes in Depression. Biol Psychiatry. 2017;81:645-53.

12. Soares-Cunha C, Coimbra B, Sousa N, Rodrigues AJ. Reappraising striatal D1- and D2-neurons in reward and aversion. Neurosci Biobehav Rev. 2016;68:370-86.

13. Gong S, Doughty M, Harbaugh $C R$, Cummins A, Hatten ME, Heintz $N$, et al. Targeting Cre recombinase to specific neuron populations with bacterial artificial chromosome constructs. J Neurosci: Off J Soc Neurosci. 2007;27:9817-23.
14. Golden SA, Covington HE 3rd, Berton O, Russo SJ. A standardized protocol for repeated social defeat stress in mice. Nat Protoc. 2011;6:1183-91.

15. Kim H-D, Call T, Carotenuto S, Johnson R, Ferguson D. Testing Depression in Mice: a Chronic Social Defeat Stress Model. Bio-Protoc. 2017;7:e2203.

16. Krishnan V, Han MH, Graham DL, Berton O, Renthal W, Russo SJ, et al. Molecular adaptations underlying susceptibility and resistance to social defeat in brain reward regions. Cell. 2007;131:391-404.

17. Kuleshov MV, Jones MR, Rouillard AD, Fernandez NF, Duan Q, Wang Z, et al. Enrichr: a comprehensive gene set enrichment analysis web server 2016 update. Nucleic Acids Res. 2016;44:W90-97.

18. Haimon Z, Volaski A, Orthgiess J, Boura-Halfon S, Varol D, Shemer A, et al. Reevaluating microglia expression profiles using RiboTag and cell isolation strategies. Nat Immunol. 2018;19:636-44.

19. Gerfen CR. The neostriatal mosaic: multiple levels of compartmental organization in the basal ganglia. Annu Rev Neurosci. 1992;15:285-320.

20. Lobo MK, Karsten SL, Gray M, Geschwind DH, Yang XW. FACS-array profiling of striatal projection neuron subtypes in juvenile and adult mouse brains. Nat Neurosci. 2006;9:443-52.

21. Heiman M, Schaefer A, Gong S, Peterson JD, Day M, Ramsey KE, et al. A translational profiling approach for the molecular characterization of CNS cell types. Cell. 2008;135:738-48.

22. Ho $H$, Both $M$, Siniard $A$, Sharma $S$, Notwell $J H$, Wallace $M$, et al. A Guide to Single-Cell Transcriptomics in Adult Rodent Brain: the Medium Spiny Neuron Transcriptome Revisited. Front Cell Neurosci. 2018;12:159.

23. Robinson JT, Thorvaldsdottir H, Winckler W, Guttman M, Lander ES, Getz G, et al. Integrative genomics viewer. Nat Biotechnol. 2011;29:24-6.

24. Kronman H, Richter F, Labonte B, Chandra R, Zhao S, Hoffman G, et al. Biology and Bias in Cell Type-Specific RNAseq of Nucleus Accumbens Medium Spiny Neurons. Sci Rep. 2019;9:8350.

25. Afgan E, Baker D, van den Beek M, Blankenberg D, Bouvier D, Cech M, et al. The Galaxy platform for accessible, reproducible and collaborative biomedical analyses: 2016 update. Nucleic Acids Res. 2016;44:W3-W10.

26. Rouillard AD, Gundersen GW, Fernandez NF, Wang Z, Monteiro CD, McDermott $M G$, et al. The harmonizome: a collection of processed datasets gathered to serve and mine knowledge about genes and proteins. Database: J Biol Databases Curation. 2016;2016:baw100.

27. Klaassen RV, Stroeder J, Coussen F, Hafner AS, Petersen JD, Renancio C, et al. Shisa6 traps AMPA receptors at postsynaptic sites and prevents their desensitization during synaptic activity. Nat Commun. 2016;7:10682.

28. Spence EF, Dube S, Uezu A, Locke M, Soderblom EJ, Soderling SH. In vivo proximity proteomics of nascent synapses reveals a novel regulator of cytoskeleton-mediated synaptic maturation. Nat Commun. 2019;10:386.

29. Peter S, Urbanus BHA, Klaassen RV, Wu B, Boele HJ, Azizi S, et al. AMPAR Auxiliary Protein SHISA6 Facilitates Purkinje Cell Synaptic Excitability and Procedural Memory Formation. Cell Rep. 2020;31:107515.

30. Wray NR, Ripke S, Mattheisen M, Trzaskowski M, Byrne EM, Abdellaoui A, et al. Genome-wide association analyses identify 44 risk variants and refine the genetic architecture of major depression. Nat Genet. 2018;50:668-81.

31. Howard DM, Adams MJ, Clarke TK, Hafferty JD, Gibson J, Shirali M, et al. Genome-wide meta-analysis of depression identifies 102 independent variants and highlights the importance of the prefrontal brain regions. Nat Neurosci. 2019;22:343-52.

32. Choi JH, Sim SE, Kim Jl, Choi DI, Oh J, Ye S, et al. Interregional synaptic maps among engram cells underlie memory formation. Science. 2018;360:430-5

33. Cao JL, Covington HE 3rd, Friedman AK, Wilkinson MB, Walsh JJ, Cooper DC, et al. Mesolimbic dopamine neurons in the brain reward circuit mediate susceptibility to social defeat and antidepressant action. J Neurosci: Off J Soc Neurosci. 2010;30:16453-8.

34. Chaudhury D, Walsh JJ, Friedman AK, Juarez B, Ku SM, Koo JW, et al. Rapid regulation of depression-related behaviours by control of midbrain dopamine neurons. Nature. 2013;493:532-6.

35. Sun H, Damez-Werno DM, Scobie KN, Shao NY, Dias C, Rabkin J, et al. ACF chromatin-remodeling complex mediates stress-induced depressive-like behavior. Nat Med. 2015;21:1146-53.

36. Walsh JJ, Friedman AK, Sun H, Heller EA, Ku SM, Juarez B, et al. Stress and CRF gate neural activation of BDNF in the mesolimbic reward pathway. Nat Neurosci. 2014;17:27-9.

37. Chandra R, Francis TC, Nam H, Riggs LM, Engeln M, Rudzinskas S, et al. Reduced Slc6a15 in Nucleus Accumbens D2-Neurons Underlies Stress Susceptibility. J Neurosci: Off J Soc Neurosci. 2017;37:6527-38.

38. Dias C, Feng J, Sun H, Shao NY, Mazei-Robison MS, Damez-Werno D, et al. Beta-catenin mediates stress resilience through Dicer1/microRNA regulation. Nature. 2014;516:51-5. 
39. Fox ME, Chandra R, Menken MS, Larkin EJ, Nam H, Engeln M, et al. Dendritic remodeling of D1 neurons by RhoA/Rho-kinase mediates depression-like behavior. Mol Psychiatry. 2020;25:1022-34.

40. Kim HD, Hesterman J, Call T, Magazu S, Keeley E, Armenta K, et al. SIRT1 Mediates Depression-Like Behaviors in the Nucleus Accumbens. J Neurosci: Off J Soc Neurosci. 2016:36:8441-52.

41. Gray JD, Rubin TG, Kogan JF, Marrocco J, Weidmann J, Lindkvist S, et al. Translational profiling of stress-induced neuroplasticity in the CA3 pyramidal neurons of BDNF Val66Met mice. Mol Psychiatry. 2018;23:904-13.

42. Gokce O, Stanley GM, Treutlein B, Neff NF, Camp JG, Malenka RC, et al. Cellular Taxonomy of the Mouse Striatum as Revealed by Single-Cell RNA-Seq. Cell Rep. 2016;16:1126-37.

43. Saunders A, Macosko EZ, Wysoker A, Goldman M, Krienen FM, de Rivera $H$, et al. Molecular Diversity and Specializations among the Cells of the Adult Mouse Brain. Cell. 2018;174:1015-30. e1016.

44. Zeisel A, Hochgerner H, Lonnerberg P, Johnsson A, Memic F, van der Zwan J, et al. Molecular Architecture of the Mouse Nervous System. Cell. 2018;174:999-1014. e1022.

45. Stahl PL, Salmen F, Vickovic S, Lundmark A, Navarro JF, Magnusson J, et al. Visualization and analysis of gene expression in tissue sections by spatial transcriptomics. Science. 2016;353:78-82.

46. Hanchate NK, Lee EJ, Ellis A, Kondoh K, Kuang D, Basom R, et al. Connect-seq to superimpose molecular on anatomical neural circuit maps. Proc Natl Acad Sci USA. 2020;117:4375-84.

47. Pei J, Grishin NV. Unexpected diversity in Shisa-like proteins suggests the importance of their roles as transmembrane adaptors. Cell Signal. 2012;24: 758-69.

48. von Engelhardt J. AMPA Receptor Auxiliary Proteins of the CKAMP Family. Int J Mol Sci. 2019;20:1460.

49. Han W, Li J, Pelkey KA, Pandey S, Chen X, Wang YX, et al. Shisa7 is a GABAA receptor auxiliary subunit controlling benzodiazepine actions. Science. 2019;366: 246-50.

50. Pignatelli M, Tejeda HA, Barker DJ, Bontempi L, Wu J, Lopez A et al. Cooperative synaptic and intrinsic plasticity in a disynaptic limbic circuit drive stress-induced anhedonia and passive coping in mice. Mol Psychiatry. 2020; https://doi.org/ 10.1038/s41380-020-0686-8. e-pub ahead of print

51. Vialou V, Robison AJ, Laplant QC, Covington HE 3rd, Dietz DM, Ohnishi YN, et al. DeltaFosB in brain reward circuits mediates resilience to stress and antidepressant responses. Nat Neurosci. 2010;13:745-52.

52. Todtenkopf MS, Parsegian A, Naydenov A, Neve RL, Konradi C, Carlezon WA Jr. Brain reward regulated by AMPA receptor subunits in nucleus accumbens shell. $J$ Neurosci: Off J Soc Neurosci. 2006;26:11665-9.

53. Reynolds SM, Berridge KC. Glutamate motivational ensembles in nucleus accumbens: rostrocaudal shell gradients of fear and feeding. Eur J Neurosci. 2003; 17:2187-200.

54. Lim BK, Huang KW, Grueter BA, Rothwell PE, Malenka RC. Anhedonia requires MC4R-mediated synaptic adaptations in nucleus accumbens. Nature. 2012;487: 183-9.

55. LeGates TA, Kvarta MD, Tooley JR, Francis TC, Lobo MK, Creed MC, et al. Reward behaviour is regulated by the strength of hippocampus-nucleus accumbens synapses. Nature. 2018;564:258-62.

56. Thompson SM, Kallarackal AJ, Kvarta MD, Van Dyke AM, LeGates TA, Cai X. An excitatory synapse hypothesis of depression. Trends Neurosci. 2015;38:279-94.

57. Francis TC, Chandra R, Friend DM, Finkel E, Dayrit G, Miranda J, et al. Nucleus accumbens medium spiny neuron subtypes mediate depression-related outcomes to social defeat stress. Biol Psychiatry. 2015;77:212-22.

58. Francis TC, Gaynor A, Chandra R, Fox ME, Lobo MK. The Selective RhoA Inhibitor Rhosin Promotes Stress Resiliency Through Enhancing D1-Medium Spiny Neuron Plasticity and Reducing Hyperexcitability. Biol Psychiatry. 2019;85:1001-10.

59. Covington HE 3rd, Lobo MK, Maze I, Vialou V, Hyman JM, Zaman S, et al. Antidepressant effect of optogenetic stimulation of the medial prefrontal cortex. J Neurosci: Off J Soc Neurosci. 2010;30:16082-90.

60. Vialou V, Bagot RC, Cahill ME, Ferguson D, Robison AJ, Dietz DM, et al. Prefrontal cortical circuit for depression- and anxiety-related behaviors mediated by cholecystokinin: role of DeltaFosB. J Neurosci: Off J Soc Neurosci. 2014; 34:3878-87.

61. Bagot RC, Parise EM, Pena CJ, Zhang HX, Maze I, Chaudhury D, et al. Ventral hippocampal afferents to the nucleus accumbens regulate susceptibility to depression. Nat Commun. 2015;6:7062.

62. Gibbons AS, Brooks L, Scarr E, Dean B. AMPA receptor expression is increased post-mortem samples of the anterior cingulate from subjects with major depressive disorder. J Affect Disord. 2012;136:1232-7.
63. Rosa ML, Guimaraes FS, Pearson RC, Del Bel EA. Effects of single or repeated restraint stress on GluR1 and GluR2 flip and flop mRNA expression in the hippocampal formation. Brain Res Bull. 2002;59:117-24.

64. Qin $\mathrm{Y}$, Karst $\mathrm{H}$, Joels $\mathrm{M}$. Chronic unpredictable stress alters gene expression in rat single dentate granule cells. J Neurochem. 2004;89:364-74.

65. Schwendt M, Jezova D. Gene expression of two glutamate receptor subunits in response to repeated stress exposure in rat hippocampus. Cell Mol Neurobiol. 2000;20:319-29.

66. Duric V, Banasr M, Stockmeier CA, Simen AA, Newton SS, Overholser JC, et al Altered expression of synapse and glutamate related genes in post-mortem hippocampus of depressed subjects. Int J Neuropsychopharmacol. 2013;16:69-82.

67. Kallarackal AJ, Kvarta MD, Cammarata E, Jaberi L, Cai X, Bailey AM, et al. Chronic stress induces a selective decrease in AMPA receptor-mediated synaptic excitation at hippocampal temporoammonic-CA1 synapses. J Neurosci: Off J Soc Neurosci. 2013;33:15669-74.

68. Toth E, Gersner R, Wilf-Yarkoni A, Raizel H, Dar DE, Richter-Levin G, et al. Age-dependent effects of chronic stress on brain plasticity and depressive behavior. J Neurochem. 2008;107:522-32.

69. Yuen EY, Wei J, Liu W, Zhong P, Li X, Yan Z. Repeated stress causes cognitive impairment by suppressing glutamate receptor expression and function in prefrontal cortex. Neuron. 2012;73:962-77.

\section{ACKNOWLEDGEMENTS}

This work was supported by grants from NIMH (R01 MH112716, to DF; R21 MH113679 to DF and SQ) and the Brain \& Behavior Research Foundation NARSAD Young Investigator Grant (\#29970, to H-DK). We thank Jean Lozach at OnRamp Bioinformatics for assistance with bioinformatic analysis and data deposition at GEO.

\section{AUTHOR CONTRIBUTIONS}

$\mathrm{H}-\mathrm{DK}, \mathrm{SQ}$, and DF designed the research, analyzed data and wrote the paper. H-DK, TC, SC, NTQ, AJS, and RJ performed behavioral tests. JW, XM, and NTQ performed electrophysiology and morphological analysis experiments. CX and JGP contributed bioinformatic analysis. H-DK performed all other experiments.

\section{COMPETING INTERESTS}

The authors declare no competing interests.

\section{ADDITIONAL INFORMATION}

Supplementary information The online version contains supplementary material available at https://doi.org/10.1038/s41380-021-01217-8.

Correspondence and requests for materials should be addressed to D.F.

Reprints and permission information is available at http://www.nature.com/ reprints

Publisher's note Springer Nature remains neutral with regard to jurisdictional claims in published maps and institutional affiliations.

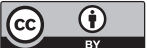

Open Access This article is licensed under a Creative Commons Attribution 4.0 International License, which permits use, sharing, adaptation, distribution and reproduction in any medium or format, as long as you give appropriate credit to the original author(s) and the source, provide a link to the Creative Commons license, and indicate if changes were made. The images or other third party material in this article are included in the article's Creative Commons license, unless indicated otherwise in a credit line to the material. If material is not included in the article's Creative Commons license and your intended use is not permitted by statutory regulation or exceeds the permitted use, you will need to obtain permission directly from the copyright holder. To view a copy of this license, visit http://creativecommons. org/licenses/by/4.0/.

(c) The Author(s) 2021 